\title{
Demografía, mercado de trabajo y tecnología: El patrón de crecimiento de Cataluña, 1970- 2020*
}

\section{MÓNICA CORREA-LÓPEZ}

Servicio de Estudios Económicos, BBVA Research, ESPAÑA y Department of Economics, University of Manchester, REINO UNIDO. E-mail: monica.correa@grupobbva.com

\author{
ANA CRISTINA MINGORANCE-ARNÁIZ \\ Departamento de Economía Aplicada, Universidad San Pablo CEU, ESPAÑA. E-mail: \\ mingor.fcee@ceu.es
}

\section{RESUMEN}

En los últimos cuarenta años, la tasa media anual de crecimiento del PIB per cápita de Cataluña se ha situado en torno al $2 \%$, resultado de un patrón de crecimiento económico que se ha desarrollado de acuerdo a dos modelos claramente diferenciados. El primero de ellos se extiende de 1970 a 1992, y en él, el crecimiento del producto potencial per cápita se apoyó, fundamentalmente, en las ganancias de productividad de la mano de obra, consecuencia del fuerte aumento de la eficiencia tecnológica. El segundo abarca el periodo 1993-2010, y con él, son la evolución favorable de los factores demográficos y del mercado laboral, especialmente el grado de utilización del factor trabajo, los que explican el crecimiento del producto potencial per cápita de Cataluña, pasando la productividad a un segundo plano al desacelerarse su crecimiento. La actual crisis económica está afectando a Cataluña con especial virulencia, frenando en gran medida su tasa de crecimiento. No obstante, a medio plazo, Cataluña podría recuperar una tasa de crecimiento del producto potencial entorno al 2,2\% siempre que se embarque en un proceso de convergencia, y cierre de la brecha tecnológica que mantiene con la economía estadounidense.

Palabras clave: Crecimiento potencial, productividad total de los factores, Cataluña.

\section{Demography, Labour Market and Technical Progress: The Pattern of Growth in Cataluña, 1970-2020}

\begin{abstract}
Over the last forty years, the pattern of long-term economic growth in Catalonia has been characterised by two very distinctive phases. During the period 1970-1992, the source of per capita output growth is found in the extensive labour productivity gains that were the result of technological efficiency improvements. The period 1993-2010, on the other hand, witnessed a favourable evolution of demographic and labour market factors - more particularly, a strong expansion of labour utilisation - while labour productivity growth slowed down substantially. This paper presents medium-term forecasts of potential growth in Catalonia and suggests that labour productivity growth may underpin an estimated potential growth rate of $2,2 \%$. Yet, the results are conditional upon Catalonia's effort at closing the technological gap with the U.S.

Keywords: Potential Growth, Total Factor Productivity, Catalonia.

Clasificación JEL: O11, O47, R11

\footnotetext{
* Las autoras agradecen a Miguel Cardoso, Rafael Doménech, Pep Ruiz Aguirre, los asistentes al seminario de economía SEE BBVA, y a los propios evaluadores anónimos de la revista de Estudios de Economía Aplicada, los comentarios y sugerencias recibidas, pues sin duda han contribuido a la mejora científica de su contenido. Además se agradece al Servicio de Estudios Económicos, BBVA, los datos facilitados. El resultado final es responsabilidad única de sus autoras. Los análisis y las conclusiones contenidas en este artículo corresponden a las autoras del mismo, y no necesariamente al Grupo BBVA.
}

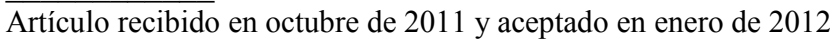

Artículo disponible en versión electrónica en la página www.revista-eea.net, ref. ə-30102 


\section{INTRODUCCIÓN}

Desde mediados de los años 70, las diferencias regionales entre los niveles de renta per cápita en España han mostrado una persistencia alta, al tiempo que la posición de las regiones en el ranking de riqueza comparada se ha mantenido muy estable. En este sentido Cataluña se ha caracterizado por ser una de las economías con mayor nivel de riqueza por habitante, tanto en comparación con el conjunto de la economía española como con el resto de Europa. La productividad de su mano de obra, el comportamiento de su mercado de trabajo, así como los rasgos de su estructura productiva, condicionan en parte, como veremos, su posición de ventaja.

Este hecho, unido a la crisis económica que se inició en el verano de 2007 afectando a las principales economías mundiales, y que cuenta entre sus consecuencias con la revisión a la baja de las expectativas de crecimiento del PIB efectivo y potencial durante los próximos años, nos lleva a preguntarnos en qué medida se verá afectada Cataluña, así como en qué modelo se ha basado hasta ahora su crecimiento económico, y si será necesario reformarlo para impulsar la recuperación y ayudarla a mantenerse en las posiciones de cabeza, así como a mejorar posiciones frente a las regiones europeas.

El objetivo esencial de este trabajo es arrojar luz sobre las consecuencias que la actual crisis económica tendrá sobre el crecimiento económico potencial de los próximos años, así como sobre los efectos que la misma debería tener sobre las decisiones de política económica en Cataluña, a fin de poder mantener las buenas posiciones que tradicionalmente Cataluña ha mostrado en términos de crecimiento y bienestar. Para ello será necesario profundizar en el análisis de su patrón de crecimiento pasado y futuro, y situar éste en perspectiva comparada con el conjunto de la economía española, lo que nos ayudará a identificar los factores subyacentes sobre los cuáles la política económica podría actuar con el objetivo de cerrar la brecha existente en el nivel de renta per cápita frente a otras áreas de su entorno. Así, nos proponemos responder, entre otros, a los siguientes interrogantes, ¿Qué factores han determinado el crecimiento económico de Cataluña? ¿Cómo se comparan éstos en relación a los factores subyacentes del crecimiento del conjunto de la economía española? ¿Se puede realizar una primera estimación de los factores que, con mayor probabilidad, se convertirán en motor del crecimiento catalán en el medio y largo plazo?

Para dar respuesta a estos interrogantes, el trabajo se organiza de la siguiente manera. En el siguiente apartado se realiza un análisis del posicionamiento de la economía catalana en términos de PIB per cápita, y se presentan los hechos estilizados de la economía catalana en comparación con otras economías de su entorno. En la tercera sección se describe la metodología empleada en la estimación del crecimiento del PIB potencial. Tras una primera revisión de las diferentes alternativas metodológicas existentes, se realiza una aproximación al 
método de estimación utilizado para dar respuesta a los interrogantes planteados. A continuación, el estudio se centra en la determinación del crecimiento potencial y en el análisis de los factores que subyacen al mismo, con una cuarta parte que comienza analizando el patrón de crecimiento de Cataluña hasta el 2010, para construir posteriormente los escenarios demográficos, laborales y tecnológicos que nos permitirán proyectar el crecimiento potencial del PIB hasta el 2020, así como conocer las contribuciones de los distintos factores al mismo.

Sólo una vez analizado el patrón de crecimiento, que muy probablemente, y salvo que se adopten cambios estructurales importantes, caracterizará a la economía catalana durante los próximos años, podremos, en la quinta parte, establecer las comparaciones oportunas entre los factores determinantes del crecimiento potencial en Cataluña y en España.

A la luz de los resultados obtenidos, el trabajo se cierra con una última sección donde se recogen las principales conclusiones, así como algunas propuestas que permitirían mejorar el crecimiento potencial de Cataluña.

\section{HECHOS ESTILIZADOS DE LA ECONOMÍA CATALANA}

Un indicador habitualmente empleado en la medición del nivel de bienestar de la economía es el producto interior bruto per cápita $(P I B / L)$ cuya evolución en Cataluña, entre 1970 y 2010 , fue positiva, registrando una tasa de crecimiento media anual del $1,8 \%$, lo que no ha supuesto en todo caso, un incremento en el poder adquisitivo de los catalanes frente a los países de su entorno ${ }^{1}$. Aún no habiendo cerrado nunca la brecha de bienestar con la UE-15,

\footnotetext{
${ }^{1}$ Tradicionalmente se ha recurrido al PIB per cápita para medir el nivel de bienestar de un país por las ventajas que presenta, y entre las que se cuentan, su simplicidad, fácil interpretación, y el hecho de que permita la comparación sincrónica y diacrónica. Así se muestra en los trabajos de Cuadrado Roura (2010), Marias Fernández et. al. (2010), Darbus y Laumann (2006), Doménech (2008), Doménech et. al. (2008), Calvo Bernardino et. al. (2011). Si bien, son muchas las críticas que han recaído sobre esta variable como indicador del bienestar, y así se dice que no es capaz de reflejar las dimensiones no económicas del bienestar dejando de lado las dimensiones sociales, ambientales, políticas y culturales; que no deduce las producciones negativas como la contaminación o el aumento de la delincuencia; que sólo recoge los medios de bienestar que son objeto de transacción mercantil, pero deja fuera los bienes y servicios producidos en el ámbito doméstico; que no tiene en cuenta el nivel de ocio del que disfrutan los ciudadanos; que no considera la desigualdad en la distribución de la renta; o que no tiene en cuenta la aplicación de políticas de desarrollo sostenible que garanticen un desarrollo a largo plazo (véase Nordhause y Tobin (1972), Somarriba-Arechavala y Pena-Trapero (2008) y Repetto et. al (1989). Fruto de estas críticas, en las últimas décadas se han desarrollado otros indicadores, generalmente sintéticos, con los que medir el bienestar de los países, así podemos hablar del Î́ndice de Bienestar Económico Sostenible de Daly y Cobb (1989) que centra su atención en la degradación del medio ambiente y la distribución de los ingresos, del Índice de Progreso Social, que tiene en cuenta no sólo aspectos económicos, sino también sociales, o del Índice de Desarrollo Humano que considera junto a aspectos económicos como el PIB per cápita, otros relacionados con la
} 
sí que ha registrado un nivel de riqueza superior al del conjunto de la economía española, que en los últimos años, y como ya se ha observado en otros periodos previos, ha cerrado la brecha que le separa de Cataluña al lograr reducir la ventaja máxima conseguida en 1996, de más de un $24 \%$, para situarla en un $13 \%$ en 2010 (véase el Gráfico 1). El mayor crecimiento demográfico de Cataluña, casi un $1 \%$ de media anual entre 1970 y 2010 , frente al poco más del $0,75 \%$ de España, es el motivo principal de ello.

\section{Gráfico 1}

Evolución del PIB per cápita relativo, UE-15 $=100$

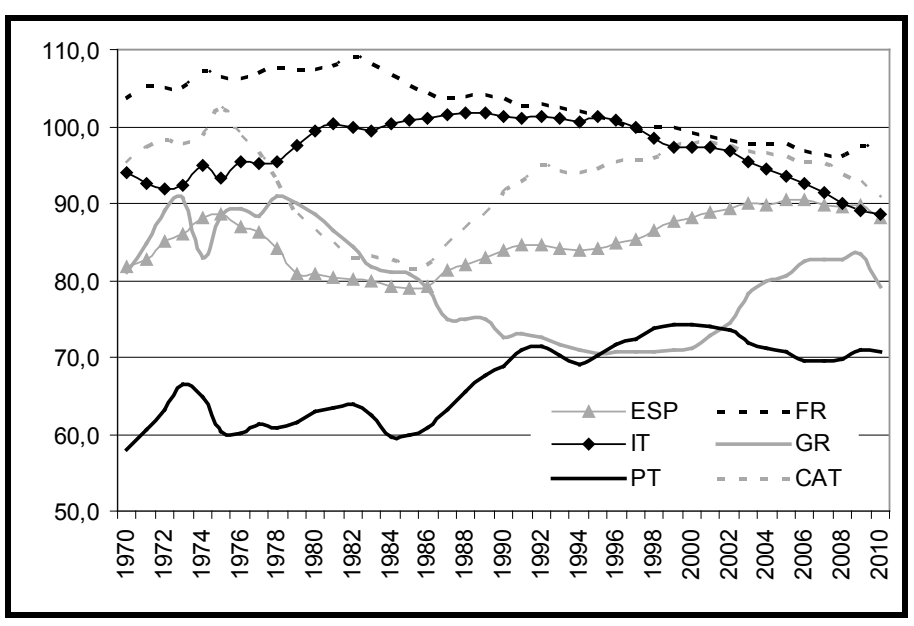

ESP (España); FR (Francia); CAT (Cataluña); PT (Portugal); GR (Grecia); IT (Italia)

Fuente: Elaboración propia con datos OCDE, BdMores e INE.

Asimismo, debe destacarse que, pese a la recesión experimentada por Cataluña en los últimos años, mayor incluso que la del conjunto de España, la economía catalana ha logrado aproximar su nivel de bienestar al de la UE-15. Así, gracias al fuerte crecimiento experimentado por la economía catalana durante la segunda mitad de los 80, alrededor de un 5,56\% entre 1986 y 1990 , se han logrado recuperar las posiciones perdidas entre mediados de los 70 y mediados de los 80, cuando el PIB per cápita de Cataluña cayó aceleradamente.

Por otro lado, hay que señalar que Cataluña disfruta de un nivel de bienestar superior al de los países europeos de la cuenca mediterránea, siendo sólo superada por Francia, que pese a todo ha perdido posiciones desde el comienzo del

salud (esperanza de vida al nacer) y la educación (tasa de alfabetización...). Para un mayor análisis de estos indicadores alternativos pueden consultarse, entre otros, los trabajos de Somarriba-Arechavala y Pena-Trapero (2008 y 2009), Pena-Trapero (2009), Phélon (2011), Sen (1999), Alvira Martín y García López (2003) y Eraukin-Iurrita (2008). 
periodo considerado, y que presenta actualmente un nivel de bienestar inferior al de la media de la UE-15, lo que ha ayudado a que a comienzos de la década pasada, Cataluña y Francia presentasen niveles de bienestar similares. En relación con Grecia cabe destacar que, aunque Cataluña disfruta de un nivel de bienestar superior, el diferencial de riqueza de más del 35\% que se registró a finales de los 90 se ha reducido hasta situarse en poco más del 6\% en 2009, diferencial que ha vuelto a aumentar como consecuencia de la fuerte recesión y las grandes incertidumbres que acechan a la economía griega. Algo parecido ocurre en el caso de Portugal, quien ha conseguido reducir su diferencial respecto a Cataluña, pasando de casi el $35 \%$ a mediados de los 90 , a poco más del $24 \%$ en el 2010. Si bien, la mayor parte de la reducción de la brecha se ha producido en los años de crisis, cuando la caída media anual ha superado el 2,75\%.

\section{Gráfico 2}

PIB per cápita medio (1970-2010) en miles de euros de 2000

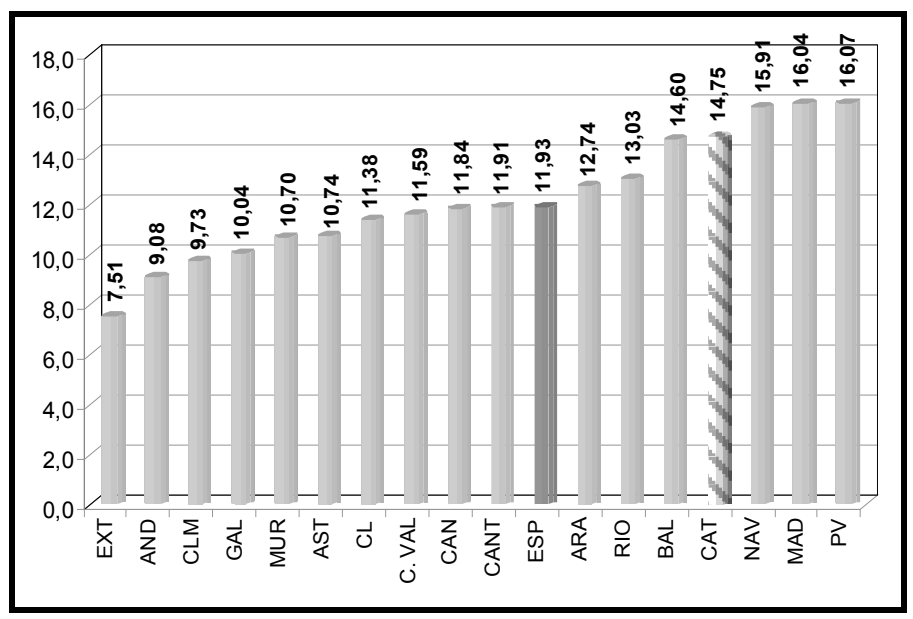

EXT (Extremadura); AND (Andalucia); CLM (Castilla La Mancha); GAL (Galicia); MUR (Murcia); AST (Asturias); CL (Castilla León); C. VAL (Comunidad Valenciana); CAN (Canarias); CANT (Cantabria); ESP (España); ARA (Aragón); RIO (La Rioja); BAL (Islas Baleares); CAT (Cataluña); NAV (Navarra); MAD (Madrid); PV (País Vasco).

Fuente: Elaboración propia con datos BdMores e INE.

Cuando la comparación se hace entre las regiones de España, Cataluña se sitúa en las primeras posiciones. Así, y si consideramos el PIB per cápita medio a lo largo del periodo comprendido entre 1970 y 2010, sólo el País Vasco, Madrid y Navarra presentan un nivel de vida medio para el periodo considerado superior al de Cataluña, tal y como se muestra en el Gráfico 2. Además, y aunque ha habido fluctuaciones en las posiciones que a lo largo de estos cuarenta años han ocupado las distintas regiones, en general las que se han mantenido en cabeza 
han sido siempre las mismas, lo mismo que ha ocurrido con las que se encuentran a la cola.

\section{MEDICIÓN DEL PRODUCTO POTENCIAL}

El PIB potencial, también llamado tendencial, constituye el mejor indicador compuesto de la oferta total de una economía. Su desviación porcentual en relación al valor efectivo del PIB permite obtener la brecha de producción, o "output gap", la cual, desde un punto de vista macroeconómico, sirve como indicador de las presiones inflacionistas.

Formalmente, el producto potencial se define como el nivel de producción sostenible obtenido a partir de la utilización de los factores de producción, esto es, el stock de capital y trabajo, en sus respectivos niveles potenciales o de equilibrio a largo plazo, combinados de acuerdo a una función de producción. La tasa de crecimiento del producto potencial establece una medida de las perspectivas de crecimiento a largo plazo de la economía. El producto potencial es una variable no observada y, en la práctica, su estimación se lleva a cabo utilizando diferentes métodos.

\subsection{La función de producción como método de estimación del PIB potencial}

Tradicionalmente, los métodos de estimación se han dividido en dos, los denominados métodos estadísticos y los basados en la estimación de relaciones estructurales. Además, en los últimos años han surgido métodos semiestructurales que, mediante filtros de carácter multivariante complementados con información económica, normalmente la curva de Phillips o la ley de Okun, resultan muy adecuados para la estimación del PIB potencial (véase Benes et al. (2010), Kuttner (1994), Staiger et al. (1997), Gerlach y Smets (1999), Orphanides y van Norden $(2000,2002)$ y Lemoine et al. (2008), entre otros). ${ }^{2}$ En la literatura se utilizan como métodos de estimación más frecuentes el filtro Hodrick-Prescott (HP), el filtro de Kalman, los modelos VAR, y el método basado en la función de producción que se sigue en muchos trabajos (véase Estrada et al. (2004), Mc Morrow y Röger (2007) o Hernández de Cos et al. (2011) entre otros).

Para la estimación del producto potencial de Cataluña, este trabajo emplea la metodología de contabilidad del crecimiento basada en la función de producción, método con supuestos basados en la teoría económica, lo que permite

\footnotetext{
${ }^{2}$ En Cotis et al. (2003), así como en Doménech y Gómez (2003), se pone de relieve que para la mayoría de países la correlación del PIB estimado bajo las diferentes metodologías se sitúa entre el 0,7 y el 0,9, reflejando que, pese a las diferencias que puedan obtenerse, los diferentes métodos de estimación describen sendas similares del PIB potencial. A conclusiones similares llegaron, para el caso de la UE, Mc Morrow y Röger (2001) y Chagny y Döpke (2001).
} 
identificar y cuantificar los hechos económicos subyacentes que explican la evolución del producto potencial. Además, bajo determinadas hipótesis factibles sobre la evolución futura de variables demográficas, de mercado de trabajo, y tecnológicas, nos permite construir escenarios alternativos sobre la posible trayectoria de la tasa de crecimiento del producto potencial a medio y a largo plazo. Finalmente, cabe destacar que, a pesar de que este método de estimación es sensible a factores cíclicos, su volatilidad es relativamente baja (véase Cotis et al. (2003)). A continuación se presenta, de forma detallada, la metodología empleada para la construcción de la tasa de crecimiento del producto potencial de Cataluña en el periodo 1977-2020.

E1 PIB per cápita se puede descomponer de acuerdo a la siguiente identidad en cada periodo del tiempo $t$ :

$$
\frac{P I B_{t}}{L_{t}} \equiv \frac{P I B_{t}}{H_{t}} \frac{H_{t}}{L_{t}^{d}} \frac{L_{t}^{d}}{L_{t}^{s}} \frac{L_{t}^{s}}{L_{t}^{15-64}} \frac{L_{t}^{15-64}}{L_{t}}
$$

en dónde el PIB per cápita se expresa como el producto de: (i) la productividad de la mano de obra por hora trabajada $(P I B / H)$, (ii) el número de horas promedio trabajadas por persona ocupada $\left(H / L^{d}\right)$ siendo $L^{d}$ el número de personas empleadas, (iii) la tasa de empleo $\left(L^{d} / L^{s}\right)$ siendo $L^{s}$ la oferta de mano de obra, (iv) la tasa de actividad $\left(L^{s} / L^{15-64}\right)$ siendo $L^{15-64}$ la población en edad de trabajar, y (v) la estructura demográfica de la población $\left(L^{15-64} / L\right)$, en dónde $L$ representa la población total. Tomando la primera diferencia del logaritmo de la expresión (1) se aproxima la identidad en función de tasas de crecimiento:

$$
\Delta \ln \frac{P I B_{t}}{L_{t}} \cong \Delta \ln \frac{P I B_{t}}{H_{t}}+\Delta \ln \frac{H_{t}}{L_{t}^{d}}+\Delta \ln \frac{L_{t}^{d}}{L_{t}^{s}}+\Delta \ln \frac{L_{t}^{s}}{L_{t}^{15-64}}+\Delta \ln \frac{L_{t}^{15-64}}{L_{t}},
$$

en dónde la tasa de crecimiento del PIB per cápita se expresa como la suma de la tasa de crecimiento de la productividad, la tasa de crecimiento de las horas promedio trabajadas, el crecimiento de la tasa de empleo, el crecimiento de la tasa de actividad, y el crecimiento de la población en edad de trabajar por habitante. La evolución temporal del PIB per cápita viene por lo tanto determinada por la evolución de los componentes que figuran en la expresión (2), de entre los cuáles únicamente la productividad puede presentar una tasa de crecimiento positiva a largo plazo. Es decir, la evolución a largo plazo de las horas promedio trabajadas, de la tasa de empleo, de la tasa de actividad, y de la estructura de la población, está acotada por sus respectivos límites (véase Doménech et al. (2008)). Aún así, cambios en la evolución a medio plazo de estas variables influirán significativamente en el perfil temporal del nivel de bienestar de la economía.

Para determinar la evolución a largo plazo del crecimiento de la productividad se supone, en primer lugar, que la producción agregada de la economía 
está adecuadamente representada por una función de producción de tipo CobbDouglas con rendimientos constantes a escala sobre capital y trabajo en cada periodo de tiempo $t$ :

$$
Y_{t}=A_{t}\left(K_{t}\right)^{\alpha}\left(h_{t} L_{t}^{d}\right)^{1-\alpha} \exp \left\{\beta S_{t}\right\}
$$

en dónde $Y$ es el PIB, $A$ es la productividad total de los factores $(P T F), K$ es el stock de capital físico productivo privado, $h$ es el número de horas promedio trabajadas por ocupado, $L^{d}$ es el número de ocupados, y $s$ es el capital humano por trabajador medido, en línea con Doménech et al. (2008), por los años promedio de escolarización ${ }^{3}$. Bajo el supuesto de que los mercados de productos y de factores operan en competencia perfecta, el parámetro $\alpha$ es la proporción del valor añadido correspondiente a las rentas del capital. Igualmente, $(1-\alpha)$ es la proporción del valor añadido correspondiente a las rentas del trabajo. Finalmente, el parámetro $\beta$ es la elasticidad del producto con respecto al capital humano ${ }^{4}$. La ecuación (3) permite obtener la senda temporal de la PTF como componente residual - el residuo de Solow - una vez que se disponen de series temporales de producción, stock de capital, horas totales trabajadas y capital humano, y se formulen supuestos factibles sobre los parámetros $\{\alpha, \beta\}$ de la función de producción.

Rescribiendo la expresión (3) en función de la relación capital-output $(K / Y)$ se obtiene la siguiente expresión de la productividad por hora trabajada:

$$
\frac{Y_{t}}{H_{t}}=\left(\frac{K_{t}}{Y_{t}}\right)^{\frac{\alpha}{1-\alpha}} A_{t}^{\frac{1}{1-\alpha}} \exp ^{\left(\beta s_{t}\right)_{1-\alpha}^{\frac{1}{1-\alpha}}}
$$

Finalmente, tomando logaritmos a la expresión (4) y primeras diferencias, la tasa de crecimiento de la productividad se expresa como:

$$
\Delta \ln \frac{Y_{t}}{h_{t} L_{t}^{d}}=\frac{1}{1-\alpha} \Delta \ln A_{t}+\frac{\alpha}{1-\alpha} \Delta \ln \frac{K_{t}}{Y_{t}}+\frac{\beta}{1-\alpha} \Delta s_{t}
$$

en dónde la tasa de crecimiento de la productividad por hora trabajada depende de la evolución del progreso técnico (PTF), del crecimiento de la ratio capitaloutput, y de la evolución de los años de escolarización.

\footnotetext{
${ }^{3}$ La forma en que tradicionalmente se presenta la función de producción no desagrega el capital humano. No obstante, la relevancia que el mismo está adquiriendo en el crecimiento de la productividad de la mano de obra y por ende del bienestar, nos lleva a considerar una función de producción en la que este factor aparezca de forma desagregada (véase Cohen y Soto (2007), De la Fuente (2005), De la Fuente y Doménech (2001a, 2001b y 2008) y Doménech (2008)).

${ }^{4}$ La elasticidad del producto respecto al capital humano $(\beta)$ es, como se reconoce en De la Fuente y Domenech (2006a), un valor aproximado, pues en la estimación se supone que un aumento del nivel educativo se obtiene mediante la escolarización total del factor trabajo, en vez de por un aumento gradual en los años de estudio de las cohortes más jóvenes.
} 
Substituyendo la expresión (5) en la expresión (2) se obtiene la descomposición de la tasa de crecimiento del PIB per cápita que se expresa, en términos de producción, como figura a continuación:

$$
\Delta \ln Y_{t}=\frac{\alpha}{1-\alpha} \Delta \ln \left(\frac{K_{t}}{Y_{t}}\right)+\frac{1}{1-\alpha} \Delta \ln A_{t}+\frac{\beta}{1-\alpha} \Delta s_{t}+\Delta \ln h_{t}+\Delta \ln \left(1-u_{t}\right)+\Delta \ln \frac{L_{t}^{s}}{L_{t}^{15-64}}+\Delta \ln \left(L_{t}^{15-64}\right)
$$

en dónde la tasa de empleo se escribe como $\left(1-u_{t}\right)$ siendo $u_{t}$ la tasa de desempleo. La tasa de crecimiento del producto potencial se estima introduciendo como inputs en la ecuación (6) la tasa de desempleo estructural (o NAIRU), el componente tendencial de la productividad total de los factores, que se extrae aplicando el filtro de Hodrick-Prescott a la serie de PTF, y los componentes tendenciales de las horas promedio trabajadas, la tasa de participación, y la población en edad de trabajar. Además, la estimación del PIB potencial requeriría incorporar los valores tendenciales del stock de capital productivo privado y de capital humano, sin embargo, en la práctica, se aproxima el valor potencial de estas variables mediante su valor observado (véase Estrada et al. (2004)).

Puesto que el objetivo de este trabajo es generar estimaciones de la tasa de crecimiento del producto potencial es necesario elaborar, previamente, escenarios alternativos del crecimiento de las diferentes variables a utilizar (ver anexo $1)$.

\section{ESTIMACIÓN Y PROYECCIÓN DEL PIB POTENCIAL}

Antes de adentrarnos en la construcción de los escenarios sobre el comportamiento de las variables demográficas, del mercado de trabajo y tecnológicas, realizaremos una primera aproximación a los factores que hasta ahora han explicado el crecimiento económico de Cataluña.

Un análisis de los factores en los que se apoya el crecimiento de la economía catalana en los últimos treinta años revela la existencia de dos períodos claramente diferenciados (véase el Cuadro 1). Por un lado, el periodo 1970-1992 que podemos dividir en dos sub-periodos, el primero entre 1970 y 1981, en el que el fuerte crecimiento de la productividad de la mano de obra $(4,11 \%)$, y, más en concreto, el de la ratio capital-output, que logra aportar el $60 \%$ del crecimiento de la productividad, se convierte en la causa esencial del crecimiento económico. No obstante, no debe minusvalorarse el impacto positivo del crecimiento de la población, mientras el empleo, fruto de la crisis que arrasa a la economía mundial, reduce el crecimiento del PIB. Y un segundo sub-periodo, 1981-1992, en el que sigue destacando el papel de la productividad de la mano de obra $(2,94 \%)$, pero ahora con un crecimiento especialmente marcado del progreso tecnológico $(1,73 \%)$ consecuencia del proceso de convergencia y "catching-up" técnico frente a los países más avanzados, mientras pierde intensidad el proceso de acumulación de capital. Además, a lo largo de este periodo, se observa una 
recuperación del mercado de trabajo, con contribuciones positivas de la tasa de empleo en los últimos años del periodo, pero también de la población en edad de trabajar, fruto del atractivo que supone para la mano de obra extranjera la recuperación económica de Cataluña, y de las mayores facilidades de acceso a su mercado de trabajo debido a la incorporación de España a la Unión Europea en 1986.

\section{Cuadro 1 \\ Descomposición de la Tasa de Crecimiento del PIB Potencial \\ (tasa media de crecimiento anual tendencial, en \%)}

\begin{tabular}{lllllllllll}
\hline & $\boldsymbol{\Delta} \mathbf{Y}$ & $\boldsymbol{\Delta} \mathrm{Y} / \mathrm{H}$ & $\boldsymbol{\Delta} \mathrm{K} / \mathrm{Y}$ & $\boldsymbol{\Delta} \mathrm{PTF}$ & $\boldsymbol{\Delta} \mathbf{S}$ & $\boldsymbol{\Delta} \mathrm{H} / \mathrm{L}^{\mathrm{d}}$ & $\boldsymbol{\Delta} \mathrm{L}^{\mathrm{d} / \mathrm{L}^{\mathrm{s}}}$ & $\boldsymbol{\Delta} \mathrm{L}^{\mathrm{s}} / \mathrm{L}^{15-64}$ & $\Delta \mathrm{L}^{15-64} / \mathrm{L}$ & $\Delta \mathrm{L}$ \\
\cline { 2 - 10 } $\mathbf{1 9 7 0 - 1 9 8 1}$ & 3,65 & 4,11 & 2,48 & 1,41 & 0,22 & $-0,68$ & $-1,25$ & $-0,12$ & 0,05 & 1,54 \\
$\mathbf{1 9 8 2 - 1 9 9 2}$ & 3,44 & 2,94 & 0,92 & 1,73 & 0,29 & $-0,47$ & $-0,27$ & 0,45 & 0,62 & 0,18 \\
$\mathbf{1 9 9 3 - 2 0 1 0}$ & 2,96 & 1,05 & 0,95 & $-0,07$ & 0,17 & $-0,33$ & 0,16 & 0,87 & 0,03 & 1,18 \\
\hline Valor medio & 3,35 & 2,70 & 1,45 & 1,03 & 0,22 & $-0,49$ & $-0,46$ & 0,40 & 0,23 & 0,97 \\
\hline
\end{tabular}

Fuente: Elaboración propia.

El segundo periodo, 1993-2010, destaca por el agotamiento experimentado en las ganancias de productividad, fundamentalmente de la PTF, y por una expansión económica apoyada en los factores demográficos y laborales. Así, mientras la tasa de actividad creció a una tasa media anual del 0,87\% entre 1995 y 2010 , la población lo hizo a una tasa de casi el 1,4\% para el mismo periodo y sólo a 6 puntos básicos menos lo hacía la población en edad de trabajar, fruto del aumento significativo de la población inmigrante, que atraída por el buen comportamiento del mercado de trabajo, creció a una tasa media anual en torno al $40 \%$ durante el periodo $2002-2007$.

Un análisis más detallado de la productividad de la mano de obra se recoge en el Gráfico 3, de donde se concluye que su comportamiento en Cataluña no dista mucho del de otros países con las excepciones de Grecia y Portugal. Así, se observa un primer periodo (1970 - 1983) de gran crecimiento, en el que la productividad de la mano de obra catalana, al igual que en el caso de España, el Área Euro 12 e Italia, se aproxima a la de la economía norteamericana, seguido de una segunda etapa (1983 - 1995) de cierta estabilidad, al menos en términos relativos frente a EE.UU. Por su parte, en la tercera fase, de 1995 a 2007, la productividad decrece, mientras que en la última fase, que se corresponde con la crisis actual, la productividad de la mano de obra catalana se recupera considerablemente al alcanzar una tasa de crecimiento media anual de casi el 3\%. 


\section{Gráfico 3}

Evolución de la productividad de la mano de obra por hora trabajada EE.UU = 100

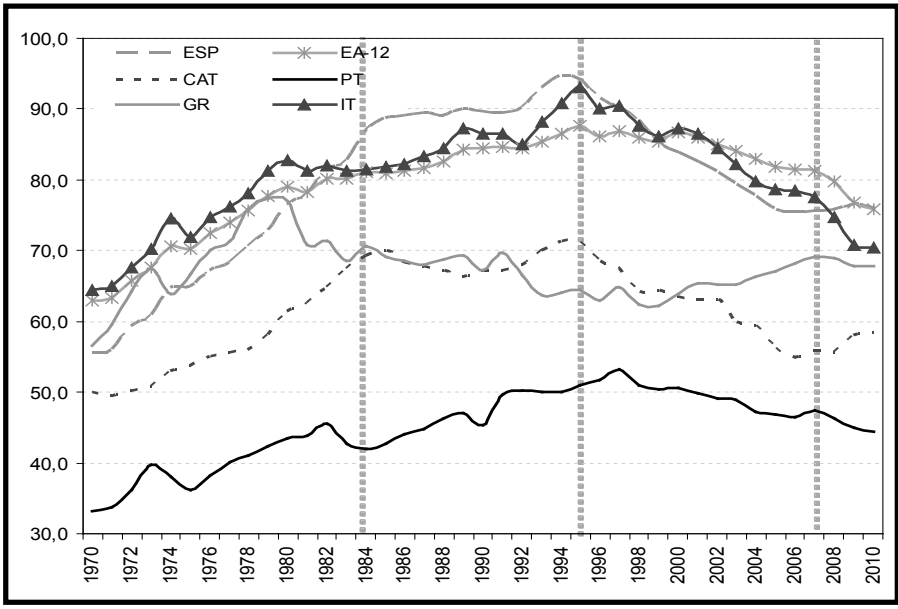

ESP (España); EA-12 (Zona Euro 12); CAT (Cataluña); PT (Portugal); GR (Grecia); IT (Italia)

Fuente: Elaboración propia con datos OCDE, BdMores e INE.

\subsection{Construcción de escenarios}

Hechas las primeras valoraciones, debemos evaluar los factores que resultarán determinantes en el crecimiento potencial de Cataluña durante los próximos años, para lo que es necesario, estimar previamente el comportamiento futuro de la tasa de crecimiento del PIB potencial. Para ello se han construido escenarios sobre el comportamiento de las variables que aparecen en la función de producción. Así, se han construido hasta tres cotas diferentes, una central que se considera la más probable, otra inferior, que de producirse conduciría a un menor crecimiento económico en el medio plazo, y una superior que permitiría alcanzar una alta tasa de crecimiento potencial.

\subsubsection{Escenarios demográficos y del mercado de trabajo}

Los escenarios de población total se han construido a partir de las proyecciones de población a corto plazo elaboradas en octubre de 2010 por el INE para el periodo 2010-2020. Para el escenario central se han empleado dichas proyecciones, mientras que para la construcción del escenario inferior se ha acudido a las proyecciones que ofrece el Instituto de Estadística Catalán (Idescat), siendo el escenario superior simétrico respecto al central. Así, la diferencia entre la tasa de crecimiento media de población en el mejor y peor de los casos se sitúa, en 2020, en los 0,39 puntos porcentuales (véase el Gráfico 4). 
Gráfico 4

Proyección de la tasa de crecimiento de la población total

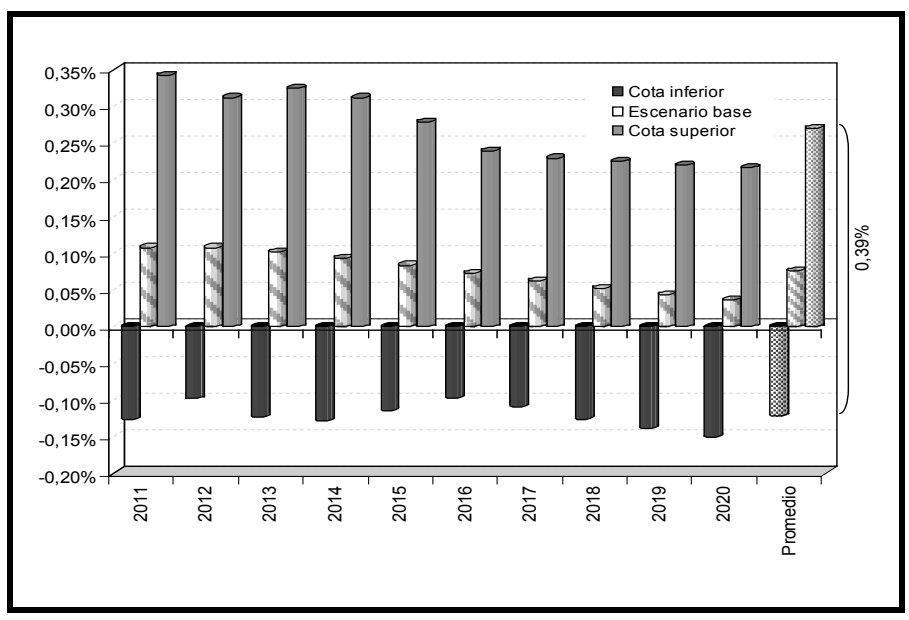

Fuente: Elaboración propia con datos del INE e Idescat.

Una vez obtenidas las tasas de crecimiento de la población total bajo cada uno de los tres escenarios, se han construido los escenarios demográficos, considerando los datos de población efectiva a 1 de Enero en cada uno de los años hasta 2010, y se han aplicado las tasas de crecimiento calculadas previamente para las previsiones hasta 2020.

Las proyecciones resultantes, recogidas en el Gráfico 5, estiman un crecimiento demográfico medio anual para el periodo $2011-2020$ de entre el $(-0,12 \%)$, en el peor de los escenarios representados, y el $0,27 \%$ en el mejor de los casos, lo que conduce a un abanico demográfico entre la cota superior e inferior en el año 2020 de 296.545 habitantes. Además, debe señalarse que el crecimiento demográfico previsto para los primeros años, tenderá a estabilizarse a partir del 2017.

Del mismo modo, y con las tasas de crecimiento que se deducen de las proyecciones a corto plazo de la población en edad de trabajar elaboradas por el INE e Idescat, se han construido los escenarios de población potencialmente activa. De este modo, la diferencia entre la tasa media de crecimiento de la cota superior e inferior para el periodo 2011-2020 se sitúa en los 0,41 puntos porcentuales.

De las proyecciones resultantes, recogidas en el Gráfico 6, se concluye que la población con edad entre 15 y 64 años se situará, muy probablemente, entre 4.728.303 habitantes en la cota inferior y 4.925.674 en la cota superior, siendo la banda de fluctuación respecto al escenario base de $+/-2,04 \%$. 
Cabe destacar la desaceleración de las tasas de crecimiento de ambas variables demográficas proyectadas a 2020, especialmente de la población en edad de trabajar, lo que se explica, en parte, por la incertidumbre que la crisis está generando en los flujos migratorios.

\section{Gráfico 5}

Proyecciones demográficas: población total

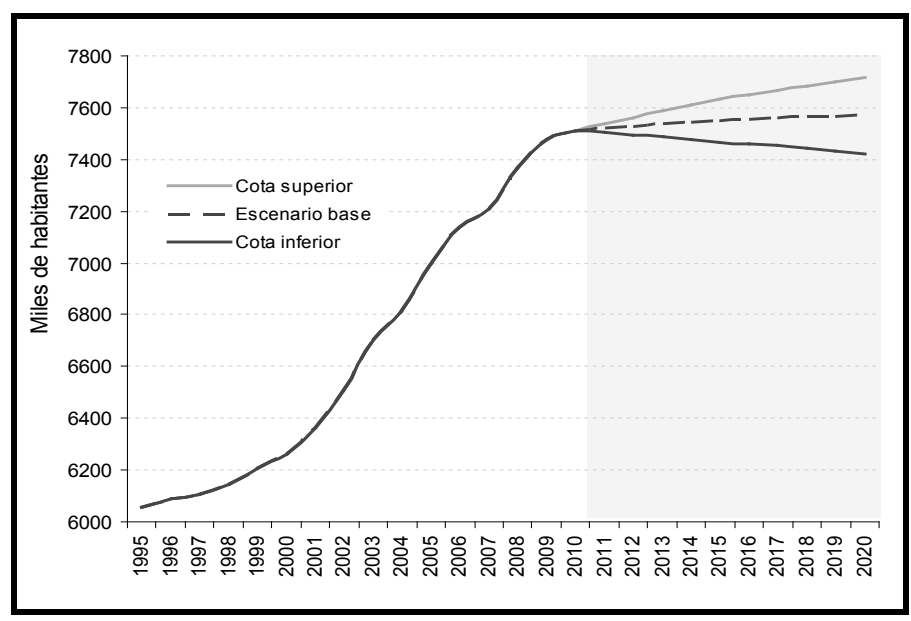

Fuente: Elaboración propia con datos BdMores, INE e Idescat.

\section{Gráfico 6}

Proyecciones demográficas: población en edad de trabajar

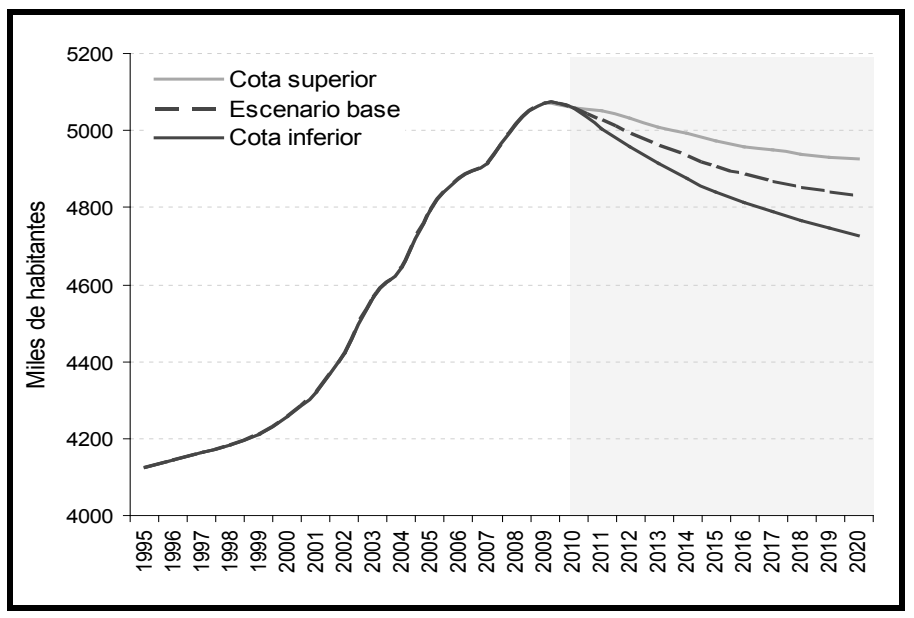

Fuente: Elaboración propia con datos INE e Idescat. 
A continuación se construyen los escenarios de las variables vinculadas al mercado de trabajo, concretamente las tasas de actividad y de paro, que nos sirven para construir los escenarios de población activa y ocupada.

Para proyectar la tasa de actividad se han empleado las estimaciones que se recogen en Ortega (2008), quién ofrece proyecciones anuales de la tasa de actividad hasta 2021. En concreto, se ha aplicado la tasa de crecimiento prevista por Ortega cada año a la tasa de actividad del 2010, lo que conduce a que en el escenario base, la tasa de actividad del 2020 alcance el 77,08\%. Para proyectar las cotas máxima y mínima, se ha sumado $+/-1,5$ puntos porcentuales al valor que toma la tasa de actividad en el último año de proyección, interpolándose de forma lineal los valores medios. Así, la variación media anual de la tasa de actividad alcanza, para todo el periodo de proyección, el valor de $0,41 \%$ en el caso de la cota superior y de $0,02 \%$ en el de la cota inferior (véase el Gráfico 7).

Con la tasa de actividad proyectada y las estimaciones de población potencialmente activa obtenidas para cada uno de los escenarios, se han construido las series de población activa del escenario base y de las correspondientes cotas hasta 2020. Los resultados conducen a una población activa en 2020 de 3.720.794 personas en el escenario base, y a una brecha para el mismo año de casi 297.000 habitantes $(+/-3,95 \%)$.

Gráfico 7

Proyecciones del mercado de trabajo: tasa de actividad

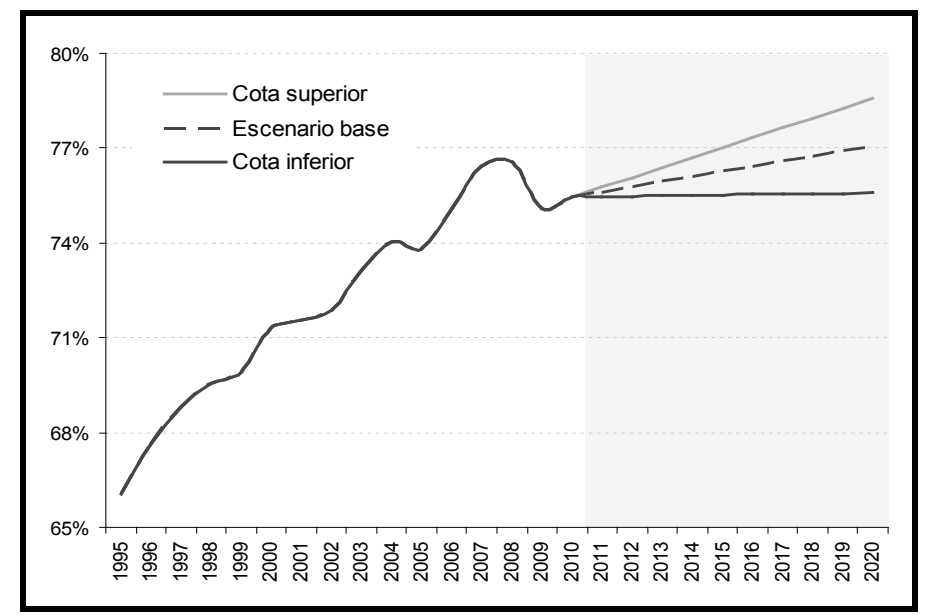

Fuente: Elaboración propia con datos IVIE, INE y Ortega (2008).

Como se observa en el Gráfico 8, y al igual que ha ocurrido con las variables demográficas, se espera una desaceleración marcada en el crecimiento de la población activa fruto de la más que probable reducción de los flujos migratorios. 
Además, la incertidumbre sobre la evolución de la población en edad de trabajar se trasladará al mercado de trabajo, y así la tasa media anual de crecimiento de la población activa para el periodo de proyección variará entre $(-0,66 \%)$ en el escenario inferior y $0,14 \%$ en el superior.

\section{Gráfico 8}

Proyecciones del mercado de trabajo: población activa

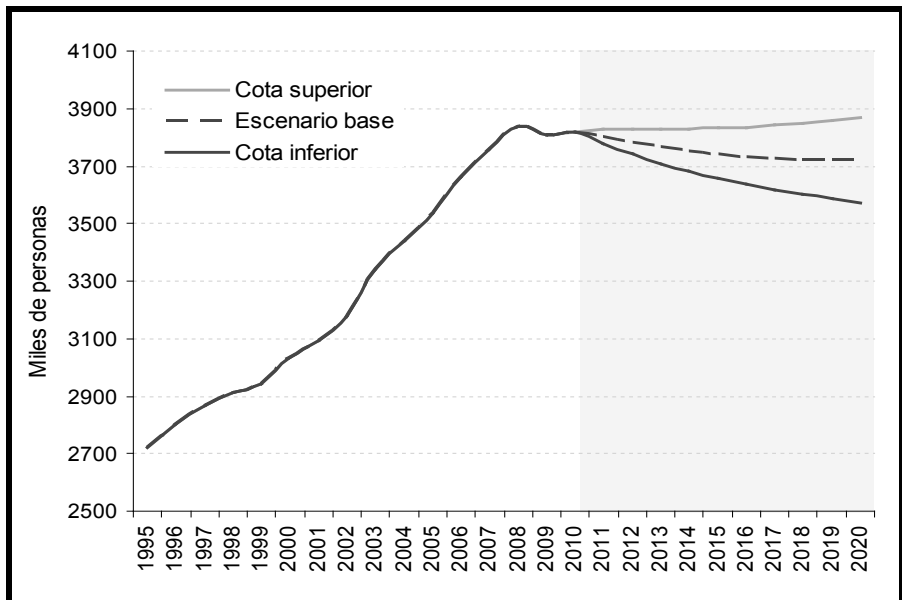

Fuente: Elaboración propia con datos IVIE, INE y Ortega (2008).

Las proyecciones de la población ocupada se han realizado a partir de la tasa de paro así como de la población activa, previamente proyectada, obteniéndose la población empleada hasta 2020 por la diferencia entre la población activa y la parada. La tasa de paro de los años 2011 y 2012 del escenario base se ha construido a partir de las previsiones elaboradas por FUNCAS (2011), lo que nos ha permitido considerar los efectos que a corto plazo tendrá la crisis económica sobre el mercado de trabajo.

Para los años siguientes se ha considerado que la tasa de paro de Cataluña convergerá al $7 \%$ en 2030 , valor de pleno empleo, y próximo al que registró antes de la crisis la Euro Zona en 2008 y no muy alejado de la tasa de paro de Cataluña en 2007 (6,55\%). Los valores medios se han interpolado linealmente.

Como se observa en el Gráfico 9, se espera que la tasa de paro crezca hasta 2012, momento en el cual empezará a descender hasta registrar, en 2020, una tasa del $13,86 \%$ en el escenario base. Pese a este fuerte crecimiento del paro, mayor que el registrado en otros períodos económicos adversos, es poco probable que llegue a alcanzarse la tasa de paro del año 1994.

Los escenarios superior e inferior se han construido a partir del escenario base, disminuyendo y aumentando la tasa de paro de éste en un $2 \%$ respectiva- 
mente en el año 2030, de modo que en el 2020 la tasa de paro sería del 12,86\% en la cota superior y del $14,86 \%$ en la cota inferior. A partir de estas tasas de paro se han podido construir los escenarios de la NAIRU, recogidos, al igual que la tasa de paro efectiva, en el Gráfico 9. La senda de la NAIRU presenta tasas de crecimiento positivas hasta 2015 , momento en que se alcanzará una tasa del $15,15 \%$ en el escenario base, situándose la variación media anual experimentada por la NAIRU en el periodo 2011 - 2020 entre el $0,54 \%$ de la cota inferior, y el $-0,60 \%$ de la superior.

Gráfico 9

Proyecciones del mercado de trabajo: tasa de paro y NAIRU

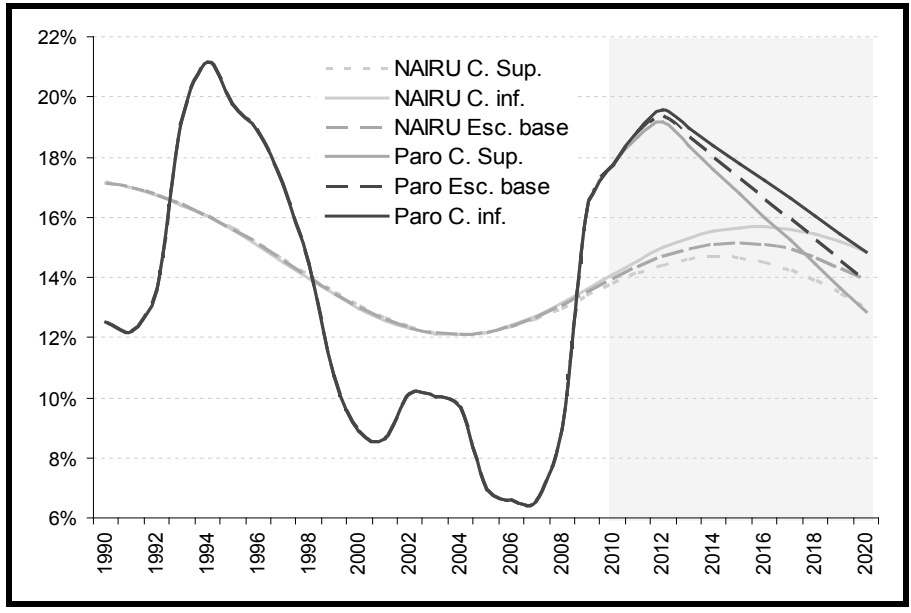

Fuente: Elaboración propia con datos IVIE, INE, Ortega (2008) y Funcas (2011).

Con la proyección de la tasa de paro es posible estimar la población ocupada para cada uno de los escenarios alternativos, y a partir del nivel de ocupación y de la población en edad de trabajar, se obtiene la tasa de empleo. La amplitud del intervalo entre la tasa de empleo de la cota superior e inferior en el último año de predicción es de $+/-2 \%$, siendo la tasa de empleo en el escenario base de $66,4 \%$ en 2020.

Finalmente, y en relación con las horas promedio trabajadas, se asume un único escenario, haciéndolas permanecer constantes en 1611 horas por año y trabajador a lo largo de todo el periodo de proyección.

\subsubsection{Capital humano: años medios de escolarización}

Para aproximar la dotación de capital humano, se ha utilizado, en línea con Doménech et al. (2008), los años medios de escolarización de la población ocu- 
pada que ofrece la serie de capital humano construida por el IVIE ${ }^{5}$. En concreto, se han empleado datos EPA, superando los inconvenientes de escasez de observaciones de los datos censales que señalan Raymond y Roig (2006) y AlonsoMeseguer y Sosvilla-Rivero (2004).

La senda central futura de los años promedio de escolarización se proyecta a partir de la siguiente ecuación estimada para el periodo 1975-2010:

$$
\Delta \ln s_{t}=0,065-0,023 \ln s_{t-1}
$$

en dónde $s_{t}$ es el número de años promedio de escolarización de la población. En el valor de estado estacionario, o largo plazo, los años de escolarización promedio de la población catalana alcanzarán la cifra de 16,92 años $^{6}$. De este modo se estima que el capital humano de Cataluña se situará en 11,90 años en 2020 , lo que supondrá una tasa media anual de crecimiento entre 2011 y 2020 de $0,94 \%$. La cota superior se obtiene sumando 0,75 a los años de escolarización del escenario central en 2030, e interpolando los valores medios, mientras que la cota inferior se ha construido como simétrica a la superior. Estos resultados, sintetizados en el Gráfico 10, muestran una cierta aceleración en la tasa de crecimiento anual, al menos, y como ya ha ocurrido en otras crisis, durante los primeros años de proyección y hasta que la recuperación económica sea una realidad. Así, dicha tasa de crecimiento pasará del $0,05 \%$ en 2010 al $0,53 \%$ en el 2020 en el escenario base.

\footnotetext{
${ }^{5}$ Aunque el promedio de los años de escolarización es la variable habitualmente empleada por la literatura para medir el capital humano, cabe destacar que ésta no incluye la formación universitaria de tercer ciclo (másters y doctorado) y los conocimientos que provienen de la formación no reglada como es la formación continua y permanente dentro de la empresa o la experiencia laboral. Además, tal y como se indica en De la Fuente y Doménech (2006a) y De la Fuente et al. (2005), no incluye el ajuste por calidad de la enseñanza, factor determinante de la productividad de la mano de obra (véase Estrada et al. (2006), Hanushek y Woessmann (2007, 2009), entre otros) y que queda recogida en la PTF.

${ }^{6}$ El crecimiento de 6 años en el número medio de años de escolarización situará el capital humano de Cataluña en el estado estacionario ligeramente por encima del nivel que presentan actualmente economías como la de Estados Unidos, Nueva Zelanda, Canadá y los países Nórdicos, quienes en 2008 mostraron un capital humano con 5 años más de formación que Cataluña (véase Swivel (2009) y Gutiérrez-Doménech (2009)).
} 


\section{Gráfico 10}

Evolución del capital humano: Años medios de escolarización

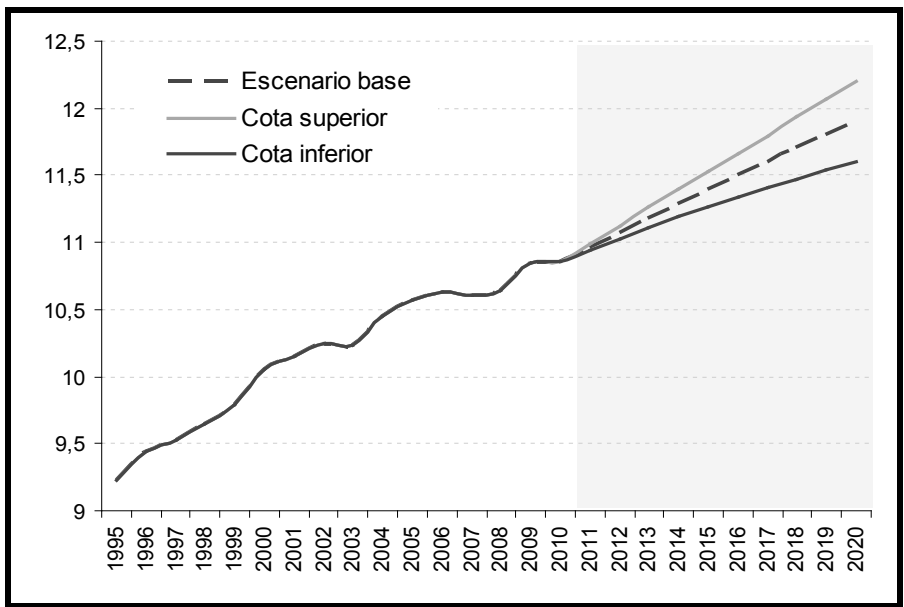

Fuente: Elaboración propia con datos IVIE.

\subsubsection{Relación Capital-Output}

En el largo plazo, la relación capital-output convergerá a su valor de estado estacionario dado por la expresión:

$$
K=\frac{I_{h p}}{g+n+\delta}
$$

en dónde $I_{h p}$ es la tasa de inversión en capital físico productivo, tanto público como privado -en particular, la inversión productiva media del periodo 2005 $2009-n$ la tasa de crecimiento del factor trabajo $-2 \%$ en el estado estacionario - $g$ la tasa de crecimiento a largo plazo combinada de la PTF y del capital humano - estimada en el $1 \%$ - y $\delta$ es la tasa de depreciación del stock de capital - estimada en el 5\% ${ }^{7}$. De esta forma, la senda central de la relación capital físico productivo-output de Cataluña alcanzará el valor de 2,45 en el estado estacio-

\footnotetext{
${ }^{7}$ Para obtener la tasa de depreciación del capital productivo se ha acudido al método de inventario permanente (metodología recomendada por el Sistema Europeo de Cuentas SEC-95) que toma como punto de partida la siguiente ecuación, estándar en la teoría económica, de acumulación de capital:

$$
K_{t+1}=(1-\delta) K_{t}+I_{t}
$$

siendo $K$ el stock de capital productivo privado, $I$ la inversión privada y $\delta$ la tasa de depreciación del capital. A partir de las series de stock de capital productivo y de inversión productiva, se puede obtener la tasa de depreciación del capital productivo para cada año. En este caso incorporamos el supuesto de que la tasa del último año se mantiene constante a partir de entonces, lo que permite estimar una tasa de depreciación del 5\% en Cataluña.
} 
nario. Por su parte, el valor de estado estacionario en la cota inferior se supone de 2,26, correspondiente al valor medio de la relación capital-output entre 2006 y 2009. Asimismo, la cota superior se ha considerado simétrica a la inferior con respecto al escenario base, lo cual implicaría una senda de convergencia a largo plazo que situaría la relación capital-output en 2,63.

\subsubsection{Productividad Total de los Factores}

La PTF recoge el conjunto de factores que determinan el progreso técnico, entre los cuáles destaca el capital tecnológico, la inversión en $\mathrm{I}+\mathrm{D}$, la calidad del capital humano, el sistema de regulación, el entorno macroeconómico, la composición sectorial de la economía y el tamaño de las empresas, y su serie se obtiene como residuo de la función de producción. Para su proyección hasta 2020 se ha adoptado el mecanismo de corrección de error descrito en De la Fuente y Doménech (2006b) y Doménech et al. (2008), tal y como se muestra en el Gráfico 11.

\section{Gráfico 11}

Crecimiento de la productividad total de los factores

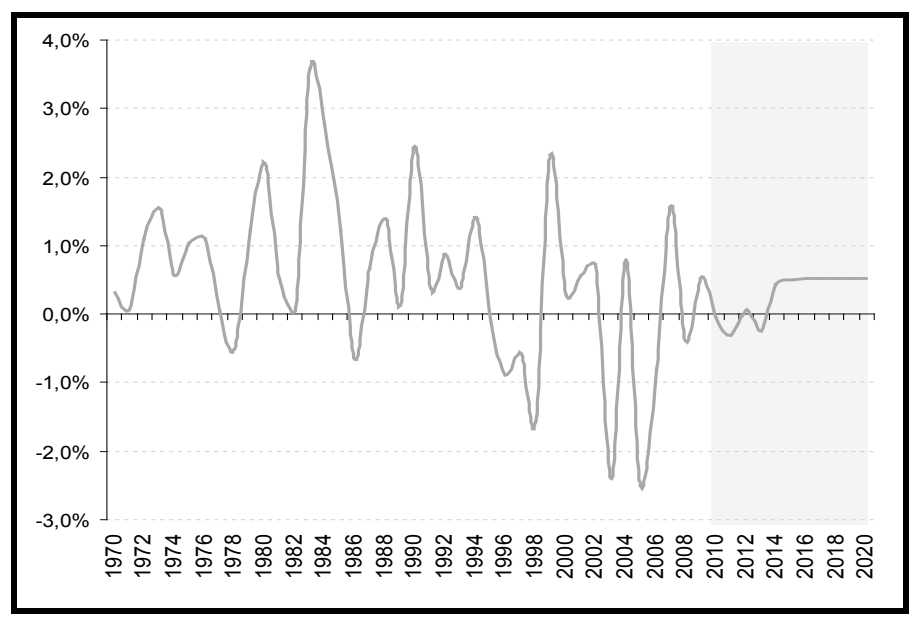

Fuente: Elaboración propia.

En concreto, la tasa de crecimiento de la PTF de Cataluña se regresa sobre su propio retardo y sobre la brecha tecnológica con respecto a Estados Unidos para el periodo 1965-2010, lo que supone la existencia de difusión internacional del conocimiento entre la economía líder y las seguidoras, tal y como se afirma en 
Jones $(2002)^{8}$. No obstante, debemos ser conscientes, como sostiene Borondo Arribas (2008), que la difusión tecnológica es más sencilla, y se obtienen mejores resultados, cuanto mayor es la apretura comercial del país imitador, más I+D se hace en el propio país, y mayor es la formación de su mano de obra, pues será capaz de asimilar mejor la tecnología extranjera.

Finalmente, en relación con los parámetros de la función de producción, se ha construido la elasticidad del producto con respecto al factor trabajo a partir de las series de remuneración del trabajo y de valor añadido bruto a precios corrientes que ofrece la base de datos BdMores. Su valor promedio a partir de 1980 se sitúa en 0,6342 , cifra que está en línea con las aproximaciones recogidas en Röger (2006), Denis et al. (2006) y McMorrow y Röger (2007) quienes lo sitúan en 0,63 para el conjunto de los Estados miembros de la Unión Europea o Doménech (2008), quien fija un valor de 0,64 para la economía española, mientras la elasticidad del producto con respecto al capital alcanza el valor de 0,3658. Por otro lado, en línea con De la Fuente y Doménech (2006a), se ha supuesto un valor de 0,0765 para la elasticidad del producto respecto al capital humano $\{\beta\}$.

\subsection{El crecimiento potencial de Cataluña, 1970-2020}

La combinación de los escenarios descritos nos va permitir construir un abanico de proyecciones alternativas para el crecimiento potencial, aumentando las probabilidades de que el mismo se sitúe dentro del intervalo establecido. Así, y como se muestra en el Gráfico 12, alrededor del escenario central aparecen, progresivamente y sombreados, los intervalos asociados a una mejor o peor evolución de las variables demográficas, del mercado laboral y tecnológicas. Así, la primera banda alrededor del escenario central corresponde al mejor y peor escenario demográfico. Sucesivamente, los intervalos de confianza se van abriendo al mejorar y empeorar la proyección anterior con los escenarios alternativos de la tasa de actividad, la tasa de desempleo estructural, los años medios de escolarización, y la ratio capital-output. De esta forma, el límite superior de

\footnotetext{
${ }^{8}$ Como sostiene Jones (2002), la economía norteamericana se ha caracterizado por presentar una tasa de crecimiento media anual del PIB per cápita estable, y ligeramente inferior al $2 \%$, en los últimos 125 años. Asimismo, Comin y Hobijn (2011) argumentan que las diferencias en la dinámica de la PTF registradas entre países de Europa Occidental y Japón durante el periodo de posguerra se debieron, en parte, a las diferencias subyacentes en los patrones de adopción tecnológica. Los países que más se beneficiaron de la asistencia técnica estadounidense experimentaron una aceleración en sus tasas de adopción, propiciando un mayor crecimiento y convergencia. Por ello, para recuperar la senda de crecimiento económico y mejorar el nivel de bienestar de Cataluña, es necesario aumentar la baja contribución al crecimiento que actualmente presenta la PTF (véase Denis et. al. (2006)) cerrando la brecha tecnológica que Cataluña mantiene con EE.UU, aumentando para ello el gasto en $\mathrm{I}+\mathrm{D}$, y participando en los procesos internacionales de difusión del conocimiento.
} 
la última banda corresponde a la mejor combinación posible de escenarios, mientras que el límite inferior del último intervalo de confianza corresponde a la peor combinación posible de los distintos escenarios.

Como puede apreciarse en el Gráfico 12, el crecimiento tendencial del PIB en la senda central se situaría en 2020 en torno al 2,24\%, con un intervalo de confianza de $+/-36$ puntos básicos $(2,60 \%$ y $1,88 \%)$. La mayor incertidumbre sobre la senda futura del crecimiento del producto potencial es fruto del comportamiento esperado en las variables demográficas, que incorporan unas bandas de fluctuación del $+/-8,6 \%$ respecto al crecimiento esperado en el escenario base. Asimismo, se observa una gran incertidumbre en la progresión futura de la tasa de actividad y, por construcción, en la capacidad de expandir el número de ocupados. En contraposición, la menor fuente de incertidumbre se encuentra en la evolución de los años medios de escolarización.

Por otro lado, es necesario destacar la reducida probabilidad conjunta de cualquiera de los dos escenarios extremos. Así, el escenario más desfavorable para la economía catalana en 2020 supondría la combinación simultánea de varios eventos adversos en comparación con el central, desde la reducción en la tasa de crecimiento de la población en edad de trabajar hasta la reducción en la relación capital-output pasando por un aumento del $0,02 \%$ en la tasa de actividad.

\section{Gráfico 12}

Proyecciones de crecimiento del PIB potencial de Cataluña 1975-2020

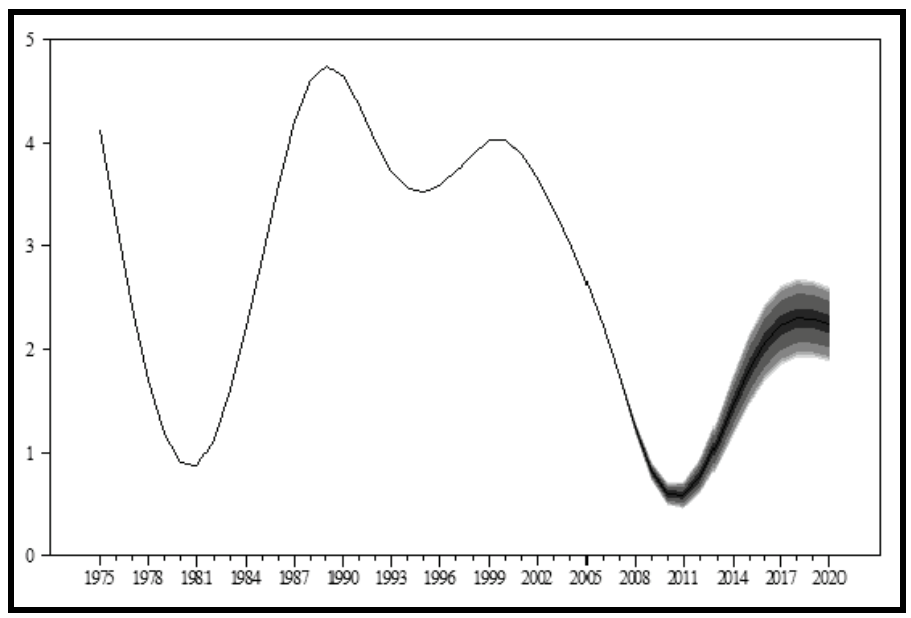

Fuente: Elaboración propia.

Desde el año 2000, Cataluña ha experimentado una importante desaceleración en su tasa de crecimiento potencial, cuya recuperación no se iniciará hasta 
principios del 2013, siendo poco probable que se retomen las tasas de crecimiento de finales de los 80 y los 90, cuando la economía catalana llegó a presentar tasas de crecimiento potencial de entre el 5\% y el $4 \%$.

Las bajas tasas de crecimiento potencial que se alcanzarán en los próximos años revelan que la actual crisis será, con gran probabilidad, la mayor que se ha vivido a lo largo de todo el periodo analizado, llegando a superar a la de 1992, o incluso a la que se vivió a finales de la década de los 70 .

\subsection{Contribuciones al crecimiento potencial}

Tan importante como conocer la tasa de crecimiento del PIB potencial, es saber cuáles son los factores en los que se sustentará el mismo, pues de este modo podremos conocer las variables sobre las que hay margen para actuar a fin de favorecer el crecimiento. En este sentido, y con respecto a los factores subyacentes que explican la senda de crecimiento potencial representada en el Gráfico 12, el Cuadro 2 muestra que, en los próximos años, una parte importante del crecimiento económico se deberá al crecimiento de la productividad de la mano de obra, que desempeñará, si cabe, un papel más destacado que en la crisis anterior. Así, entre 1993 y 2010, la productividad de la mano de obra explicó en torno al $35 \%$ del crecimiento potencial del periodo, mientras que, a 2020, la proporción del crecimiento potencial que vendrá explicada por la evolución de la productividad de la mano de obra ascenderá al $52 \%$, muy por encima de las aportaciones del resto de variables.

Por otro lado, cabe destacar que, para el conjunto del periodo de predicción, sólo la estructura demográfica $\left(\mathrm{L}^{15-64} / \mathrm{L}\right)$ y las horas medias trabajadas presentan contribuciones negativas, mientras que entre el resto de variables cabe destacar las aportaciones del progreso tecnológico y de la tasa de empleo, pues entre las dos cubrirán algo más del $60 \%$ del crecimiento del PIB.

\section{Cuadro 2}

Descomposición de la Tasa de Crecimiento del PIB Potencial de Cataluña

(tasa media de crecimiento anual tendencial, en \%)

\begin{tabular}{|c|c|c|c|c|c|c|c|c|c|c|}
\hline & $\Delta Y$ & $\Delta \mathrm{Y} / \mathrm{H}$ & $\Delta K / Y$ & $\Delta$ PTF & $\Delta \mathrm{S}$ & $\Delta H / L^{d}$ & $\Delta L^{d} / L^{s}$ & $\Delta L^{\mathrm{s}} / \mathrm{L}^{15-64}$ & $\Delta \mathrm{L}^{15-64} / \mathrm{L}$ & $\Delta \mathrm{L}$ \\
\hline $1970-1981$ & 3,65 & 4,11 & 2,48 & 1,41 & 0,22 & $-0,68$ & $-1,25$ & $-0,12$ & 0,05 & 1,54 \\
\hline 1982-1992 & 3,44 & 2,94 & 0,92 & 1,73 & 0,29 & $-0,47$ & $-0,27$ & 0,45 & 0,62 & 0,18 \\
\hline $1993-2010$ & 2,96 & 1,05 & 0,95 & $-0,07$ & 0,17 & $-0,33$ & 0,16 & 0,87 & 0,03 & 1,18 \\
\hline \multicolumn{11}{|l|}{ 2011-2020 } \\
\hline Senda Central & 1,68 & 0,87 & 0,22 & 0,52 & 0,14 & $-0,15$ & 0,52 & 0,19 & $-0,10$ & 0,35 \\
\hline Senda Superior & 1,93 & 0,82 & 0,33 & 0,33 & 0,16 & $-0,12$ & 0,53 & 0,26 & $-0,33$ & 0,75 \\
\hline Senda Inferior & 1,43 & 0,98 & 0,17 & 0,70 & 0,11 & $-0,16$ & 0,50 & 0,13 & $-0,14$ & 0,12 \\
\hline
\end{tabular}

Fuente: Elaboración propia. 
Así mismo, llama la atención la variación en la contribución de la PTF en función del escenario ante el que nos encontremos, así, en el mejor de los casos, y debido a la mayor contribución del resto de factores, el progreso tecnológico reduce la suya en casi 20 puntos básicos, ocurriendo justo lo contrario cuando nos situamos en la peor de las alternativas posibles.

\section{Cuadro 3}

Descomposición de la Tasa de Crecimiento del PIB Potencial de Cataluña en el periodo de proyección en diferentes subperiodos

(tasa media de crecimiento anual tendencial, en \%)

\begin{tabular}{|c|c|c|c|c|c|c|c|c|c|c|}
\hline Senda Central & $\Delta \ln Y$ & $\Delta \ln (\mathrm{Y} / \mathrm{H})$ & $\Delta K / Y$ & $\Delta$ PTF & $\Delta \mathrm{S}$ & $\Delta \ln (H / L d)$ & $\Delta \ln ($ Ld/Ls) & $\Delta \ln \left(L s / L^{15-64}\right)$ & $\Delta \ln \left(\mathrm{L}^{15-64} / \mathrm{L}\right)$ & $\Delta \ln (\mathrm{L})$ \\
\hline 2011-2013 & 0,81 & 1,35 & 1,06 & 0,18 & 0,11 & $-0,26$ & $-0,69$ & 0,19 & $-0,46$ & 0,68 \\
\hline 2014-2016 & 1,78 & 0,83 & 0,16 & 0,53 & 0,15 & $-0,13$ & 0,66 & 0,24 & $-0,28$ & 0,45 \\
\hline 2017-2020 & 2,27 & 0,55 & $-0,36$ & 0,76 & 0,14 & $-0,08$ & 1,32 & 0,16 & 0,31 & 0,02 \\
\hline Senda Superior & $\Delta \ln Y$ & $\Delta \ln (\mathrm{Y} / \mathrm{H})$ & $\Delta \mathrm{K} / \mathrm{Y}$ & $\Delta$ PTF & $\Delta \mathrm{s}$ & $\Delta \ln (H / L d)$ & $\Delta \ln (\mathrm{Ld} / \mathrm{Ls})$ & $\Delta \ln \left(L s / L^{15-64}\right)$ & $\Delta \ln \left(L^{15-64} / L\right)$ & $\Delta \ln (\mathrm{L})$ \\
\hline 2011-2013 & 0,92 & 1,14 & 1,11 & $-0,10$ & 0,13 & $-0,25$ & $-0,65$ & 0,28 & $-0,42$ & 0,81 \\
\hline 2014-2016 & 2,03 & 0,77 & 0,27 & 0,33 & 0,18 & $-0,09$ & 0,68 & 0,32 & $-0,44$ & 0,80 \\
\hline 2017-2020 & 2,61 & 0,63 & $-0,21$ & 0,66 & 0,18 & $-0,04$ & 1,31 & 0,21 & $-0,18$ & 0,68 \\
\hline Senda Inferior & $\Delta \ln Y$ & $\Delta \ln (\mathrm{Y} / \mathrm{H})$ & $\Delta K / Y$ & $\Delta$ PTF & $\Delta \mathrm{S}$ & $\Delta \ln (H / L d)$ & $\Delta \ln ($ Ld/Ls) & $\Delta \ln \left(L s / L^{15-64}\right)$ & $\Delta \ln \left(\mathrm{L}^{15-64} / \mathrm{L}\right)$ & $\Delta \ln (\mathrm{L})$ \\
\hline 2011-2013 & 0,69 & 1,59 & 1,03 & 0,46 & 0,10 & $-0,27$ & $-0,74$ & 0,10 & $-0,47$ & 0,48 \\
\hline 2014-2016 & 1,51 & 0,96 & 0,11 & 0,73 & 0,12 & $-0,13$ & 0,64 & 0,16 & $-0,11$ & 0,00 \\
\hline 2017-2020 & 1,92 & 0,53 & $-0,43$ & 0,86 & 0,10 & $-0,09$ & 1,33 & 0,12 & 0,09 & $-0,07$ \\
\hline
\end{tabular}

Fuente: Elaboración propia.

Un análisis más detallado del periodo de predicción, dividido ahora en subperíodos, se recoge en el Cuadro 3. De dicho Cuadro se extrae que, durante los primeros años, el mercado de trabajo contribuirá negativamente al crecimiento económico, pues la tasa de actividad será incapaz de compensar la contribución negativa de la tasa de empleo. En promedio, el mercado de trabajo volverá a mostrar contribuciones positivas a partir de 2013, conducido por el proceso de reabsorción del exceso de oferta de mano de obra generada por la recesión económica. Por su parte, la contribución al crecimiento de la población en edad de trabajar en relación a la población total sólo alcanzará valores positivos en el último sub-periodo, con la única excepción de la senda superior en la que el crecimiento esperado en la población total será siempre superior al de la población con edades comprendidas entre 15 y 64 años.

Por otro lado, la contribución de la productividad de la mano de obra irá disminuyendo con el tiempo, debido al proceso de descapitalización que se vivirá en los próximos años fruto de la coyuntura económica adversa por la que se atraviesa, las dificultades para compensar la falta de inversión privada con in- 
versión pública debido al elevado endeudamiento y a los problemas para acceder a la financiación.

No obstante, el comportamiento del capital humano, y sobre todo del progreso tecnológico, resultarán fundamentales para evitar que la productividad de la mano de obra pueda llegar a contribuir negativamente al crecimiento del PIB. Ambas variables mostrarán tasas de variación crecientes, ahora bien, mientras a partir del 2017, el crecimiento del capital humano será cada vez menor, el de la PTF se intensificará durante los próximos años, en busca de mejoras de competitividad, los recursos se dirijan a sectores y empresas más productivas y se inicie un proceso de convergencia con el líder tecnológico.

\section{CATALUÑA Y ESPAÑA: COMPARATIVA A MEDIO PLAZO}

Una rápida comparación entre la senda descrita por el crecimiento potencial de Cataluña y España nos lleva a afirmar que ambas áreas, tal y como era de esperar, han tenido comportamientos similares a lo largo del periodo de análisis. Así, y como se observa en el Gráfico 13, las recesiones económicas han afectado a ambas economías, si bien, mientras la primera de los años 70 lo hizo con menor intensidad sobre España que sobre Cataluña, la segunda, de comienzos de los 90, tuvo efectos similares en ambas zonas. Además, y con la única excepción del periodo comprendido entre 1987 y 1996, la economía nacional siempre ha mostrado un crecimiento potencial superior al de Cataluña.

Por otro lado, y desde el año 2000, ambas áreas se han visto sometidas a un proceso de recesión que se ha reflejado en unas tasas de crecimiento cada vez menores, con caídas más intensas en el caso de la economía española que en el de Cataluña. La comparación entre ambas tasas de crecimiento conduce a afirmar que Cataluña, muy probablemente, liderará el proceso de recuperación económica debido al carácter exportador de su economía.

Un análisis detallado de las contribuciones de los diferentes factores determinantes del crecimiento del PIB potencial se muestra, para Cataluña, en el Gráfico 14 y, para España, en el Gráfico 15. De la comparación de ambos Gráficos se deduce, en primer lugar, que tanto Cataluña como España presentan una tasa de crecimiento potencial promedio, a lo largo del periodo considerado, en torno al $3 \%$, al tiempo que su perfil temporal resulta muy similar. Sin embargo, los factores que han contribuido a este comportamiento presentan intensidades diferentes en uno y otro caso, especialmente en el caso de la productividad de la mano de obra y de las variables demográficas. 


\section{Gráfico 13}

Crecimiento potencial de Cataluña y España, escenario base

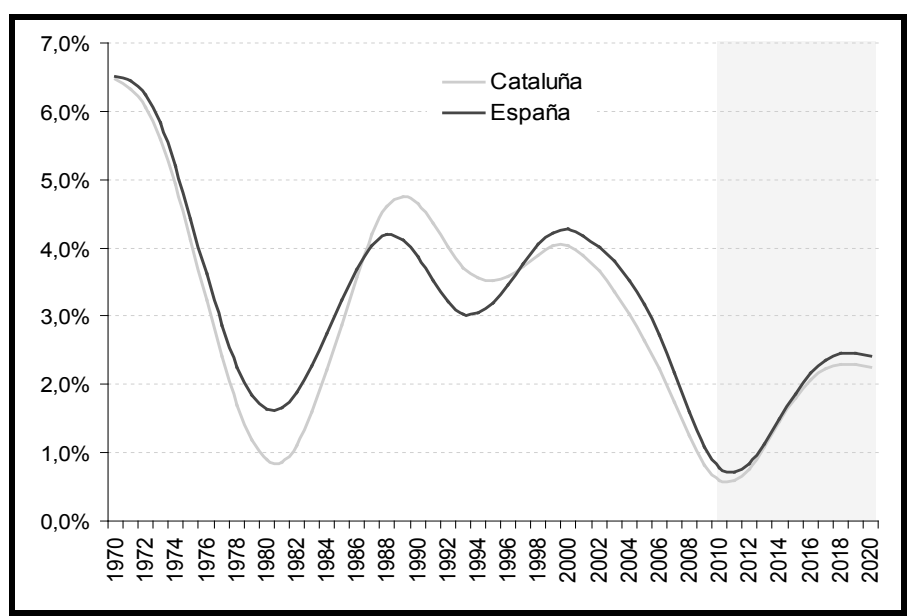

Fuente: Elaboración propia.

Gráfico 14

Contribuciones al crecimiento potencial de Cataluña, escenario base

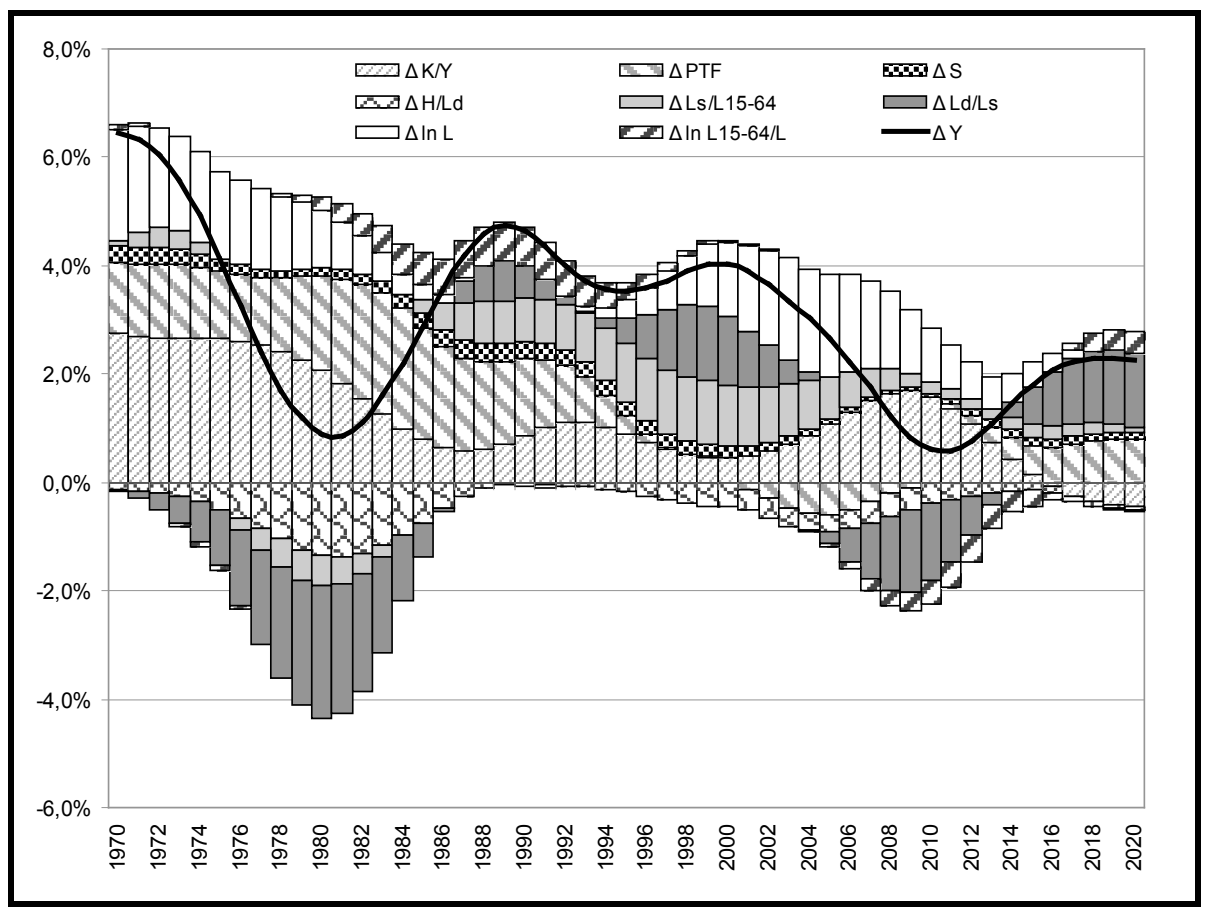

Fuente: Elaboración propia. 
Gráfico 15

Contribuciones al crecimiento potencial de España, escenario base

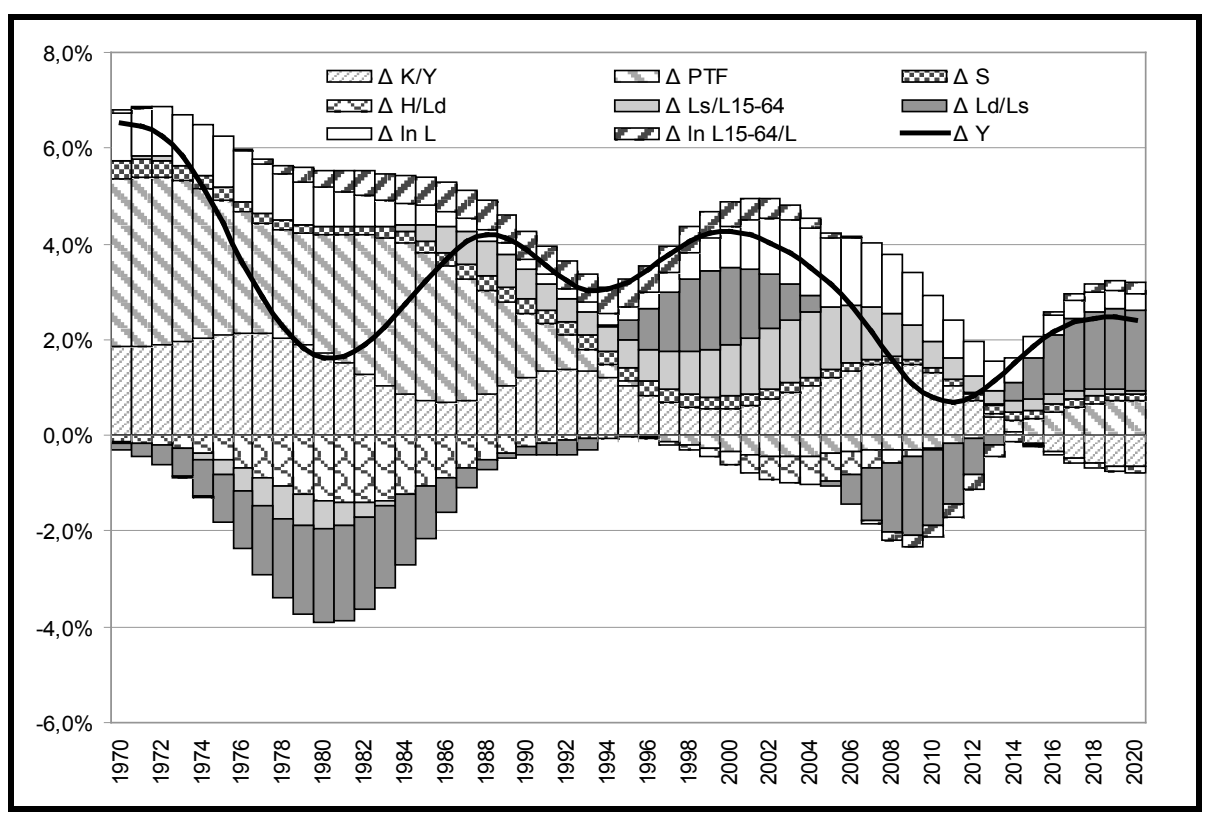

Fuente: Elaboración propia.

Así, si bien el progreso tecnológico ha llegado a tener contribuciones negativas al crecimiento en ambos casos, en el de Cataluña lo ha hecho con menor intensidad y durante un periodo de tiempo más breve. No obstante, la contribución media de la PTF al crecimiento es inferior en Cataluña, especialmente cuando se excluye el periodo de proyección, lo que se explica por el patrón de especialización catalán, que tiende a apoyarse en sectores de tecnología baja (construcción y turismo) o tecnología media (automoción), permitiéndose así la absorción de la creciente mano de obra en edad de trabajar (véase Canals y Fernández (2008) $)^{9}$. Por su parte, la contribución de la ratio capital-output, ha sido en general más intensa en Cataluña que en España. El proceso de capitalización que llevó a cabo la economía catalana durante los años 70, una economía de marcado carácter industrial que requería fuertes inversiones, se convirtió en ele-

\footnotetext{
${ }^{9}$ La OCDE clasifica cada industria según el contenido tecnológico de los bienes producidos. En primer lugar se encuentran las industrias de alto contenido tecnológico, incluyendo los sectores relacionados con la aeronáutica, así como algunas industrias de los sectores farmacéutico, de equipos de comunicación, ordenadores y maquinaria de oficina, y de motores. En segundo lugar se encuentran las industrias de contenido tecnológico medio que incluyen, entre otros, parte de los sectores relacionados con la producción de vehículos, maquinaria y el sector químico. Finalmente, los alimentos, bebidas, tabaco y zapatos son sectores de contenido tecnológico bajo.
} 
mento decisivo de este hecho. Tanto el comportamiento de la PTF, como el de la ratio capital-output, resultan claves a la hora de explicar la mayor contribución de la productividad de la mano de obra de Cataluña - el 52\% para todo el periodo de proyección frente al $31 \%$ en el caso de España -.

En cuanto a las variables demográficas, cabe señalar que la mayor contribución al crecimiento que tradicionalmente ha caracterizado a la población total pasará a un segundo plano durante el periodo de proyección. El freno que para la inmigración ha supuesto la crisis económica se convierte en una baza decisiva, y así, frente a una contribución media anual del $0,54 \%$ en el caso de España, en Cataluña ésta sólo alcanzará el 0,35\%.

\section{Cuadro 4}

Descomposición de la Tasa de Crecimiento del PIB Potencial de Cataluña y España (tasa media de crecimiento anual tendencial del PIB, en \%)

\begin{tabular}{|c|c|c|c|c|c|c|c|c|c|c|}
\hline & $\Delta Y$ & $\Delta \ln (Y / H)$ & $\Delta K / Y$ & $\Delta$ PTF & $\Delta \mathrm{s}$ & $\Delta \mathrm{H} / \mathrm{Ld}$ & $\Delta \mathrm{Ld} / \mathrm{Ls}$ & $\Delta \mathrm{Ls} / \mathrm{L}_{15-64}$ & $\Delta \mathrm{L}^{15-64} / \mathrm{L}$ & $\Delta \mathrm{L}$ \\
\hline \multicolumn{11}{|l|}{ Cataluña } \\
\hline $1970-1980$ & 3,65 & 4,11 & 2,48 & 1,41 & 0,22 & $-0,68$ & $-1,25$ & $-0,12$ & 0,05 & 1,54 \\
\hline 1981-1992 & 3,44 & 2,94 & 0,92 & 1,73 & 0,29 & $-0,47$ & $-0,27$ & 0,45 & 0,62 & 0,18 \\
\hline $1993-2010$ & 2,96 & 1,05 & 0,95 & $-0,07$ & 0,17 & $-0,33$ & 0,16 & 0,87 & 0,03 & 1,18 \\
\hline 2011-2020 & 1,68 & 0,87 & 0,22 & 0,52 & 0,14 & $-0,15$ & 0,52 & 0,19 & $-0,10$ & 0,35 \\
\hline Valor medio & 2,93 & 2,25 & 1,14 & 0,90 & 0,20 & $-0,41$ & $-0,21$ & 0,35 & 0,15 & 0,81 \\
\hline \multicolumn{11}{|l|}{ España } \\
\hline $1970-1980$ & 4,04 & 5,05 & 1,93 & 2,86 & 0,26 & $-0,69$ & $-1,10$ & $-0,31$ & 0,12 & 0,97 \\
\hline $1981-1992$ & 3,35 & 3,52 & 1,01 & 2,23 & 0,28 & $-0,73$ & $-0,76$ & 0,40 & 0,58 & 0,33 \\
\hline $1993-2010$ & 3,25 & 1,02 & 1,01 & $-0,20$ & 0,22 & $-0,27$ & 0,34 & 0,96 & 0,32 & 0,88 \\
\hline 2011-2020 & 1,70 & 0,52 & 0,06 & 0,30 & 0,16 & $-0,05$ & 0,47 & 0,25 & $-0,04$ & 0,54 \\
\hline Valor medio & 3,09 & 2,53 & 1,00 & 1,30 & 0,23 & $-0,44$ & $-0,26$ & 0,32 & 0,25 & 0,68 \\
\hline
\end{tabular}

Fuente: Elaboración propia.

Finalmente, y en relación al comportamiento de las variables vinculadas al mercado de trabajo cabe señalar que éste es bastante similar en ambas zonas, debiendo destacarse la contribución positiva, aunque reducida, de la tasa de actividad, así como las contribuciones negativas que en media presentan la tasa de empleo y las horas trabajadas. No obstante, como ha ocurrido ya en recesiones previas, una parte importante de la recuperación económica se sustentará en el crecimiento del empleo, que mostrará contribuciones positivas a lo largo de todo el periodo de proyección. 
A modo de resumen, y como se muestra en el Cuadro 4, las contribuciones de cada una de las variables al crecimiento potencial presentan los mismos signos en ambas regiones, y aunque si bien existen diferencias en las intensidades cuando la comparación se realiza por periodos, éstas se diluyen cuando se consideran los valores medios del periodo global comprendido entre 1970 y 2020.

\section{CONCLUSIÓN}

En los últimos cuarenta años, el patrón de crecimiento de la economía catalana se ha desarrollado de acuerdo a dos modelos de crecimiento claramente diferenciados. Durante el periodo 1970-1992, el crecimiento potencial se apoyó, fundamentalmente, en las ganancias de productividad de la mano de obra, propiciadas en un primer momento por el fuerte proceso de capitalización, y en un segundo periodo por el mayor crecimiento de la PTF. Alternativamente, durante el periodo 1993-2010, el crecimiento potencial catalán vino explicado por la evolución favorable de los factores demográficos y del mercado de trabajo, más concretamente, por la fuerte expansión en la tasa de actividad. A medio plazo, las perspectivas presentadas en este trabajo indican que, con alta probabilidad, la tasa de crecimiento potencial de Cataluña será más baja que la registrada a lo largo de los años previos, consecuencia de la caída de inversión, el reajuste del sector inmobiliario, y la mayor incertidumbre a la que se enfrenta la economía catalana en relación a la evolución de la población en edad de trabajar y la tasa de actividad.

Adicionalmente, el trabajo recoge un posible cambio del patrón de crecimiento de la economía catalana, que se sustentaría de nuevo en el crecimiento de la productividad, y más en concreto del progreso tecnológico. Sin embargo, el estudio también revela que este patrón de crecimiento será factible únicamente si la economía catalana se embarca en un proceso de convergencia con la economía estadounidense. En este sentido, la crisis económica que actualmente se vive, aunque negativa para el empleo y el crecimiento económico, debe ser considerada como una buena oportunidad para repensar la estructura productiva, así como los factores sobre los que se apoyará el crecimiento futuro.

Para conseguir tal objetivo, se perfila como necesario el crear un entorno macroeconómico estable, caracterizado por el funcionamiento eficiente de los mercados de factores, y desarrollar una política de incentivos que favorezca, en última instancia, la mejora de la calidad del capital humano, la inversión privada, y la innovación empresarial tanto en aspectos organizativos como en $\mathrm{I}+\mathrm{D}+\mathrm{i}$, lo que resultará, tal y como pusieron de manifiesto Röger et al. (2008) y Gual et al. (2006), esencial para promover la expansión del progreso tecnológico. Así, las políticas de incremento de la competencia en los mercados de bienes cobran relevancia. 


\section{REFERENCIAS BIBLIOGRÁFICAS}

ALONSO-MESEGUER, J. Y SOSVILLA-RIVERO, S. (2004), "El capital humano en España: una estimación del nivel de estudios alcanzado", Documento de trabajo de FEDEA $n^{\circ}$ 2004-08, mayo 2004.

ALVIRA MARTÍN, F. R. Y GARCÍA LÓPEZ, J. (2003), "Calidad de vida en las Comunidades Autónomas", Cuadernos de Información Económica, $n^{\circ} 173$, pp. 145-151.

BENES, J.; CLINTOS, K.; GARCÍA-SALTOS, R.; JOHNSON, M.; LAXTON, D.; MANCHEV, P. Y MATHESON, T. (2010), "Estimating potential output with a multivariate filter", Working paper of IMF $n^{\circ} 10 / 285$, Fondo Monetario Internacional.

BORONDO ARRIBAS, C. (2008), "La innovación en la literatura reciente del crecimiento endógeno", Revista Principios, $n^{\circ} 12$, pp. 11-42.

CALVO BERNARDINO, A; MINGORANCE ARNÁIZ, A. C.; BARRUSO CASTILLO, B. Y CALDERÓN PATIER, C. (2011), "la necesaria recuperación del catching-up tecnológico en el modelo de crecimiento económico andaluz", Revista de Estudios Regionales, $n^{\circ}$ 91, pp. 17-44.

CANALS, C. Y FERNÁNDEZ, E. (2008), "Luces y sombras de la competitividad exterior de España", Documentos de economía la Caixa $n^{\circ} 10$. La Caixa.

COHEN, D. Y SOTO, M. (2007), "Growth and human capital: good data, good results", Journal of Economic Growth, $n^{\circ} 12(1)$, pp. 51-76.

COMIN, D.A. Y HOBIJN. B. (2011), "Technology Diffusion and Postwar Growth”, NBER Macroeconomics Annual 2010, The MIT Press.

COTIS, J. P.; ELMESKOV, J. Y MOUROUGANE, A. (2003), "Estimates of potential output: benefits and pitfalls from a policy perspective", Documento de la OECD del 21 de enero de 2003, OECD.

CUADRADO ROURA, J. R. (Dir) (2010), "El crecimiento económico", en Política Económica, elaboración, objetivos e instrumentos, pp. 203-241.

CHAGNY, O. Y DÖPKE, J. (2001), "Measures of the output gap in the eurozone: an empirical assessment of selected methods", Working paper of Kiel Institute $n^{\circ}$ 1053, junio 2001.

DABÚS, C. Y LAUMANN, Y. (2006), "Determinantes del crecimiento: evidencia comparada de países con diferente nivel de desarrollo", Estudios de Economía Aplicada, vol 24(1), pp. 165-180.

DALY, H. E. Y COBB, J. B. (1989), For the Common Good: Redirecting the Economy toward Community, the Environment, and a Sustainable Future, Ed. Beacon Press, Massachusetts.

DE LA FUENTE, A. (2008), "Series enlazadas de algunos agregados económicos regionales, 1995-2007", Documento de trabajo de la Dirección General de Presupuestos del Ministerio de Hacienda $n^{\circ}$ 2008-03. Ministerio de Hacienda. 
DE LA FUENTE, A. (2005), "La educación en las regiones españolas: algunas cifras preocupantes", Documento de trabajo de la Dirección General de Presupuestos del Ministerio de Hacienda $n^{\circ}$ 2005-05. Ministerio de Hacienda

DE LA FUENTE, A., DOMÉNECH, R. (2008), "Human capital, growth and inequality in the Spanish regions", en U. Stierle; M. Stierle; F. Jennings y A. Kuah, (edit), Regional Economic Policy in Europe. Challenges for Theory, Empirics and Normative Interventions, Ed. Edward Elgar, pp. 15-44

DE LA FUENTE, A., DOMÉNECH, R. (2006a), "Capital humano, crecimiento y desigualdad en las regiones españo-las", Moneda y crédito $n^{\circ} 222, p p .13-$ 78.

DE LA FUENTE, A., DOMÉNECH, R. (2006b), "Human capital in growth regressions: how much difference does data quality make?", Journal of the European Economic Association vol. 4 (1), pp. 1-36.

DE LA FUENTE, A., DOMÉNECH, R. (2001a), "Schooling data, technological diffusion and the neoclassical model", American Economic Review, 91(2), pp. 323-27.

DE LA FUENTE, A., DOMÉNECH, R. (2001b). "Educational attainment in the OECD, 1960-90", CEPR Discussion Pa-per, $n^{\circ} .3390$.

DE LA FUENTE, A., DOMÉNECH, R., JIMENO, J. F. (2005), "Capital humano, crecimiento y empleo en las regiones españolas", Documento de Economía de la Fundación Caixa Galicia.

DENIS, C.; GRENOUILLEAU, D.; MC MORROW, K.; RÖGER, W. (2006), "Calculating potential growth rates and output gaps: a revised production function approach", Economic papers $n^{\circ} 247$, Comisión Europea.

DOMÉNECH, R. (2008), "La evolución de la productividad en España y el capital humano", Documento de trabajo $n^{\circ}$ 141/2008, Laboratorio de Alternativas.

DOMÉNECH, R.; ESTRADA, A. Y GONZÁLEZ-CALBET, L. (2008), "El potencial de crecimiento de la economía española", en J. Velarde y J. M. Serrano (coord.), La España del Siglo XXI.

DOMÉNECH, R. Y GÓMEZ, V. (2003), "Estimating potencial output, core inflation and NAIRU as latent variables", Documento de trabajo de la Dirección General de Presupuestos del Ministerio de Hacienda $n^{\circ}$ 2003-06. Ministerio de Hacienda.

ESTRADA, A.; HERNÁNDEZ DE COS, P.; JAREÑO, J. (2004), "Una estimación del crecimiento potencial de la economía española", Documento Ocasional del Banco de España $n^{\circ} 0405$.

ESTRADA, A.; PONS, A.; VALLÉS, J. (2006), "La productividad de la economía española: una perspectiva internacional", Información Comercial Española $n$ 829, marzo-abril 2006, pp. 7-25. 
ERAUKIN-IURRITA, I. (2008), "The sources of economic growth in the Basque Country, Navarre and Spain during the period 1986-2004”, Investigaciones Regionales, $n^{\circ}$ 12, pp. 35-58.

GERLACH, S. Y SMETS, F. (1999), "Output gaps and monetary policy in the EMU area1”, European Economic Review vol. 43 (4-6), pp. 801-812.

GUAL, J.; JÓDAR ROSELLO, S. Y RUIZ POSINO, A. (2006), "El problema de la productividad en España. ¿Cuál es el papel de la regulación?”, Documento de economía la Caixa $n^{\circ} 01$. La Caixa.

GUTIÉRREZ-DOMÉNECH, M. (2009), "Factores determinantes del rendimiento educativo: el caso de Cataluña", Documentos de economía la Caixa $n^{\circ} 15$. La Caixa.

HANUSHEK, E. A. Y WOESSMANN, L. (2009), "Do Better Schools Lead to More Growth? Cognitive Skills, Economic Outcomes, and Causation," Working paper of NBER $n^{\circ} 14633$, NBER.

(2007). "The role of education quality for economic growth," Working paper de la serie Policy Research of World Bank n 4122, Banco Mundial.

HERNÁNDEZ DE COS, P.; IZQUIERDO, M. Y URTASAN, A. (2011), "Una estimación del crecimiento potencial de la economía española", Documentos Ocasionales del Banco de España $n^{\circ} 1104$, Banco de España.

JONES, CH. I. (2002), "Sources of U.S. economic growth in a world of ideas", American Economic Review vol. 92 (1), pp. 220-239.

KUTTNER, K. N. (1994), "Estimating Potential Output as a Latent Variable", Journal of Business \& Economic Statistics, American Statistical Association, vol. 12, $n^{\circ} 3$, pp. 361-68.

LEMOINE, M.; MAZZI, G. L.; MONPERRUS-VERONI, P.; REYNES, F. (2008), "Real time estimation of potential output and output gap for the euro-area: comparing production function with unobserved components and SVAR approaches", Paper of MPRA $n^{\circ} 13128$, Munich Personal RePEc Archive.

MARÍAS FERNÁNDEZ, P.; MARTÍNEZ ROGET, F. Y NOVELLA, S. (2010), "Bienestar económico regional: un enfoque comparativo entre regiones españolas e italianas", Investigaciones Regionales, $n^{\circ} 8$, pp. 5-36.

MC MORROW, K. Y RÖGER, W. (2007), "La estimación y los usos del concepto de tasa de crecimiento potencial: Perspectiva general de la metodología de la función de producción que utiliza la Comisión Europea", Papeles de Economía Española $n^{\circ} 111$, pp 80-98.

MC MORROW, K. Y RÖGER, W. (2001), "Potential output: measurement methods, "new" economy influences and scenarios for 2001-2010. A comparison of the UE-15 and the US", ECFIN Economic Papers $n^{\circ} 150$. Comisión Europea.

NORDHAUS, W. Y TOBIN, J. (1972), Is growth obsolete?, Ed. Columbia University Press, New York. 
ORPHANIDES, A. Y VAN NORDEN, S. (2002), "The unreliability of output gap estimates in real time", Review of economics and statistics vol. $84, n^{\circ} 4$, pp. 569-583.

ORPHANIDES, A. Y VAN NORDEN, S. (2000), "The reliability of output gap estimates in real time", Paper presentado al Econometric Society World Congress $n^{\circ} 0768$, Econometric Society.

ORTEGA, L. (2008), "Una proyección de tasas de actividad por Comunidades Autónomas 2006-2021", Documento de trabajo del Instituto Nacional de Estadística 3/08, septiembre 2008.

PENA-TRAPERO, B. (2009), "La medición del Bienestar social: una revisión crítica", Estudios de Economía Aplicada, vol 27(2), pp. 299-324.

PHÉLON, M. C. (2011), "Revisión de índices e indicadores de desarrollo. Aportes para la medición del buen vivir", Obets, Revista de Cienciasles, vol 6(1), pp. 69-95.

RAYMOND, J. L. Y ROIG, J. L. (2006), "La dotación de capital humano en la economía española", Información Comercial Española $n^{\circ}$ 829, marzo-abril 2006, pp. 67-91.

REPETTO, R.; MAGRATH, W.; WELLS, M.; BEER, C. Y ROSSINI, F. (1989), Wasting Assets, Natural Resources in the National Income Accounts, Ed. World Resources Institute, Washington.

RÖGER, W. (2006), "The production function approach to calculating potential growth and output gaps estimates for member states and the US", DGECFIN abril 2006. Comisión Europea.

RÖGER, W., VARGA, J. Y VELD, J. (2008), "How to close the productivity gap between the US and Europe: A quantitative assessment using a semiendogenous growth model", Paper presentado a la 5th DG ECFIN Annual Research Conference de la Comisión Europea (Boosting growth and productivity in an open Europe: The role of international flows of goods, services, capital and labour) celebrado los días 16/10/2008 - 17/10/2008. (http://ec.europa.eu/economy_finance/events/2008/20081016/roger_p.pdf)

SEN, A. (1999), Development as freedom, Ed. Oxford University Press, Oxford.

STAIGER, D.; STOCK, J. Y WATSON, M. (1997), "The NAIRU, Unemployment, and Monetary Policy", Journal of Economic Perspectives, 11 (1), Winter 1997, pp. 33-49.

SOMARRIBA-ARECHAVALA,N. Y PENA-TRAPERO, B. (2009), "La medición de la calidad de vida en Europa, el papel de la información subjetiva", Estudios de Economía Aplicada, vol 27(2), pp. 373-396.

SOMARRIBA-ARECHAVALA,N. Y PENA-TRAPERO, B. (2008), "Quality of life and subjective welfare in Europe, and econometrics analysis", Applied Econometrics and International Development, vol 8(2), pp. 55-68.

SWIVEL (2009), página web (http://www.swivel.com). 


\section{Anexo 1}

\section{Datos utilizados y fuentes estadísticas (1977-2008)}

Población total: Se ha obtenido de la serie histórica de población, construida a partir del Padrón Municipal a 1 de enero, que ofrece BdMores, lo que nos permite acceder a una serie continua desde 1964 hasta 2009, mientras que el dato del 2010 se obtiene del Padrón.

La población en edad de trabajar se ha obtenido, para el periodo 1971-1997, de las estimaciones intercensales de población que ofrece el INE. La serie se ha extendido hasta 2010 con datos del Padrón, y los datos previos hasta 1964 se han obtenido del IVIE.

Las variables relacionadas con el mercado de trabajo (población activa, parada y ocupada) se han tomado del IVIE, mientras que las correspondientes tasas se han calculado a partir de los datos obtenidos.

Las horas medias trabajadas se obtiene, para el periodo 1975-2010, de la serie horas medias al año trabajadas por ocupado que ofrece Idescat. La serie se ha extendido hasta 1964 con los datos que para el conjunto de la economía española ofrece la OCDE.

La serie de capital humano se ha obtenido del IVIE, quién ofrece los años medios de escolarización, bajo el sistema educativo LGE, de la población ocupada.

Para la obtención del stock de capital productivo privado en relación al PIB ha sido necesario construir primero la serie de inversión productiva así como la del PIB, ambas a precios constantes del año 2000. El PIB, entre 1980 y 2010 se ha tomado de la contabilidad regional del INE, y se ha recalculado hasta 1964 a partir de la serie de valor añadido a precios constantes que ofrece De la Fuentes (2008). Por su parte, la serie de inversión y de stock de capital físico productivo se han obtenido de la Fundación BBVA, quien ofrece series homogéneas, entre 1964 y 2008, del valor de mercado de la inversión productiva real y del stock productivo real. Esta última serie se ha extendido hasta el 2010 a través del método de inventario permanente, suponiendo la tasa de depreciación del capital productivo total en el $5 \%$. 
